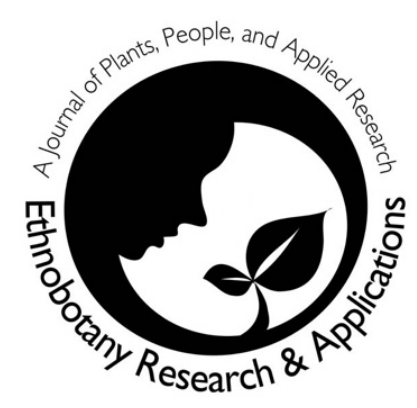

\title{
A quantitative medico- botanical expedition of Fairy Meadows National Park, Diamir, Gilgit Baltistan, Pakistan
}

\author{
Rahmat Wali, Khalid Rahman, Naveed Iqbal Raja, \\ Rahmatullah Qureshi, Rainer W. Bussmann, Zia-ur- \\ Rehman Mashwani
}

\section{Databases and Inventories}

\begin{abstract}
Background: This study was conducted to investigate the ethnobotanical knowledge of the population of the Fairy Meadow National Park, Diamir, Gilgit Baltistan. The study area was previously ignored due to physical barriers, and remoteness. The use of medicinal plants for various maladies, known to the elders of the community and passed orally to the younger generation was documented.
\end{abstract}

Methods: A total of 146 informants were interviewed using semi-structured questionnaires. The data was quantitatively analyzed employing frequency of citation (FC), use value (UV), relative frequency of citation (RFC) along with Pearson correlation coefficient (PCC).

Results: A total of 90 species belonging to 77 genera and 49 different families has been documented. These medicinal plants are used against 55 diseases, especially stomach problems (23.3\%), cough $(17.7 \%)$, asthma and fever (16.6\%). 31 out of 90 plants species displayed either novel use report (28 plants) or first time reported medicinal plants (3 plants). The first time reported medicinal plants include Allium gilgitensis, Astragalus gilgitensis and Pedicularia flava. Most of the documented plants were wild $(86 \%)$ and herbs $(60 \%)$, and leaves $(27 \%)$ were the most widely used parts. The common method of preparation was powder $(27 \%)$ mainly administered orally $(81.7 \%)$. The highest use values were found for Berberis lyceum (5.47), Thymus linearis (5.07) and Rhododendron anthopogon (5.0), while the plants with greater relative frequency of citation were Berberis lyceum (0.97), Thymus linearis (0.89) and Rhododendron anthopogon (0.75). The Pearson correlation coefficient is 0.836 between RFC and UV showing high positive association.

Conclusion: We documented a wealth of traditional knowledge and could record the uses of various species for the first time from Pakistan. The novel uses reports and first time reported medicinal plants encourage us to explore more about the hidden treasures of medicinal plants to be exploited for the wellbeing of humans.

Keywords: Ethnobotany, Medicinal Plants, Ethnic Groups, Fairy Meadow, Diamir, Gilgit Baltistan

\section{Correspondence}

Rahmat Wali' ${ }^{1}$, Khalid Rahman ${ }^{2}$, Naveed Iqbal Raja1, Rahmatullah Qureshi', Rainer W. Bussmann $^{3}$, Zia-ur-Rehman Mashwani ${ }^{{ }^{*}}$

${ }^{1}$ Department of Botany, PMAS Arid Agriculture University, Rawalpindi, 46300, Pakistan

2Department of Plant Sciences, Quaid-i-Azam University, Islamabad, Pakistan

${ }^{3}$ Department of Etnnobotany, Institute of Botany, Ilia State University, Tbilisi, Georgia

*Corresponding author: zia.botany@gmail.com, mashwani@uaar.edu.pk

Ethnobotany Research \& Applications 18:35 (2019) 


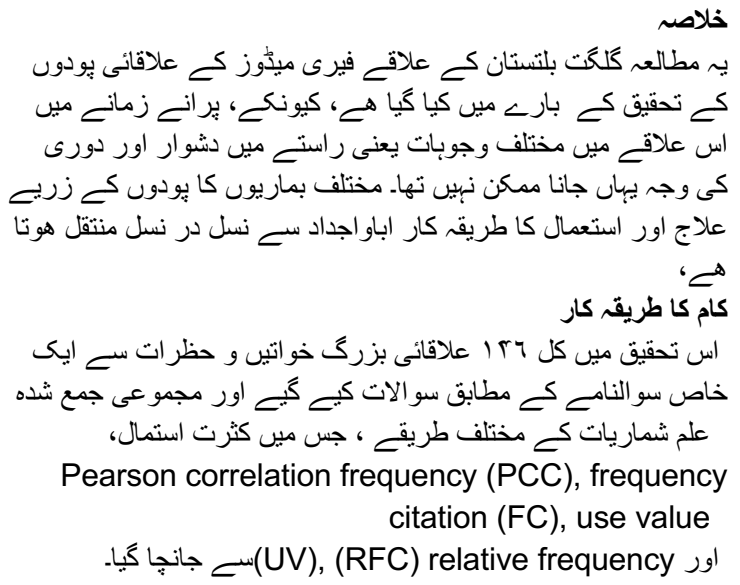

Astragalus gilgitensis and Pedicularia flava-

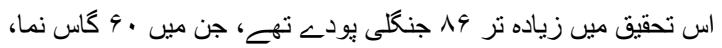

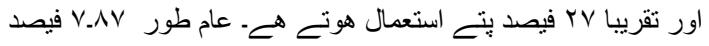

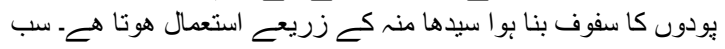

$$
\begin{aligned}
& \text { سبـ زياده استعمال }
\end{aligned}
$$

Berberis lyceum (5.47), Thymus lineari s(5.07) and

Rhododendron anthopogon (5.0), اور كثرت استعال Berberis lyceum (0.97), Thymus linearis (0.89) and Rhododendron anthopogon (0.75).

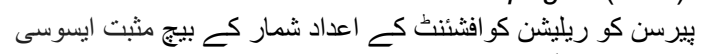
ايشن نوث كياكيا.

$$
\begin{aligned}
& \text { نتيجم اخذ شده }
\end{aligned}
$$

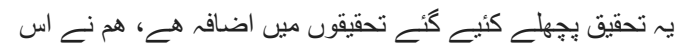

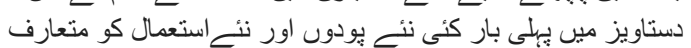

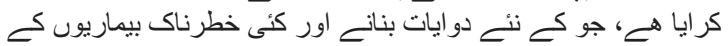

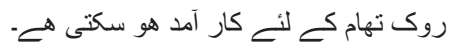

\section{Background}

Plants from the start are being used for the welfare of human and animals (Amara et al., 2017). Traditional tribal peoples harbor rich data about the utilization of plants or plant parts as medicine and food (Rehman et al., 2015; Ahmad et al., 2015). The knowledge of the therapeutic qualities of plants have a deep root and passes generation after generations among these tribal communities. It has been reported that about 400-600 plants are being used as an ethnomedicine in Pakistan (Hamayun, 2005;
Shinwari and Qaiser, 2011; Nasir et al., 1972; Muhammad et al., 2005).

Gilgit Baltistan (GB) is a region of prominent strategic and geographic importance, forming a geo-corridor exploited at times by various forces and empires, and borders contemporary powers like China, Russia and India. Gilgit Baltistan has an extraordinarily varied landscape due to the meet up of three different mountainous ranges includes Himalayas, Karakoram and Hindu Kush. Few ethnobotanical studies have been conducted in these ranges (Shinwari and Gilani, 2003). Fairy Meadow was constantly ignored because of its remoteness, difficult geology and high elevation for outsiders. The local population gives much significance to plants for their livelihood and medicinal purposes, and thus there was an urgent need to document the medicinal flora and associated traditional knowledge of this area. Such ethnobotanical inventories can provide the starting point for the development of new drugs (Bibi et al., 2011; Shinwari et al., 2013; Umadevi et al., 2013). This study constitutes the first endeavor to explore the study area ethnobotanically with focus on medicinal plants.

\section{Materials and Methods}

The present study was carried out during June 2015 to September 2016. In this period the study area was visited several times in different growing seasons of the medicinal plants.

\section{Study area (Geo-ethnography and Socio- economic conditions of the area)}

The study area is a natural alpine meadow at the base of Nanga Parbat, which is located at a latitude of $35^{\circ} \mathrm{N}$ and longitude of $74^{\circ} \mathrm{E}$. From the Karakorum Highway $(\mathrm{KKH})$, this region is situated towards the south side at a short distance of $15 \mathrm{~km}$, and $443 \mathrm{~km}$ from Islamabad, the capital of Pakistan (Fig. 1). The area is called "Fairy Meadow," which originated from the German climber Willy Merkl in 1930, after his unsuccessful endeavor to achieve the summit of Nanga Parbat (Khan, 1997). However, the local name is "Fantori," which means "the place where moustaches bearing people live." Fairy Meadow is popular for its lofty magnificence and is the heart of the northern region of Pakistan. It is also called "Paradise on Earth" because of the incomparable beauty it possesses. The area has been a major attraction for nature lovers as well as researchers, climbers and photographers for a very long period. The rich and lush green forests around fairy meadow provide an excellent habitat for wildlife. The study area comprised of many small villages have human settlements namely Tatu, Jail, Phongtori, Fairy 
Meadow, Bayal Camp, Bathrait, Bezar and Veterare. Diamir is pre-dominantly a hilly area with difficult terrains, make it physically isolated from the main areas of the country. This area also comprised of narrow valleys and high mountainous peaks. This physical situation of the area forces the inhabitant to rely on the local resources for food and other basic life necessities include health. The flora of the Diamir district is very diverse, with many medicinal plants and hundreds of other economically important species. Local communities mainly depend upon wild plant resources for their variety of economic purposes. The local livelihood strategy has shifted over time from hunting to agriculture, animal husbandry, forestry, trade and tourism.

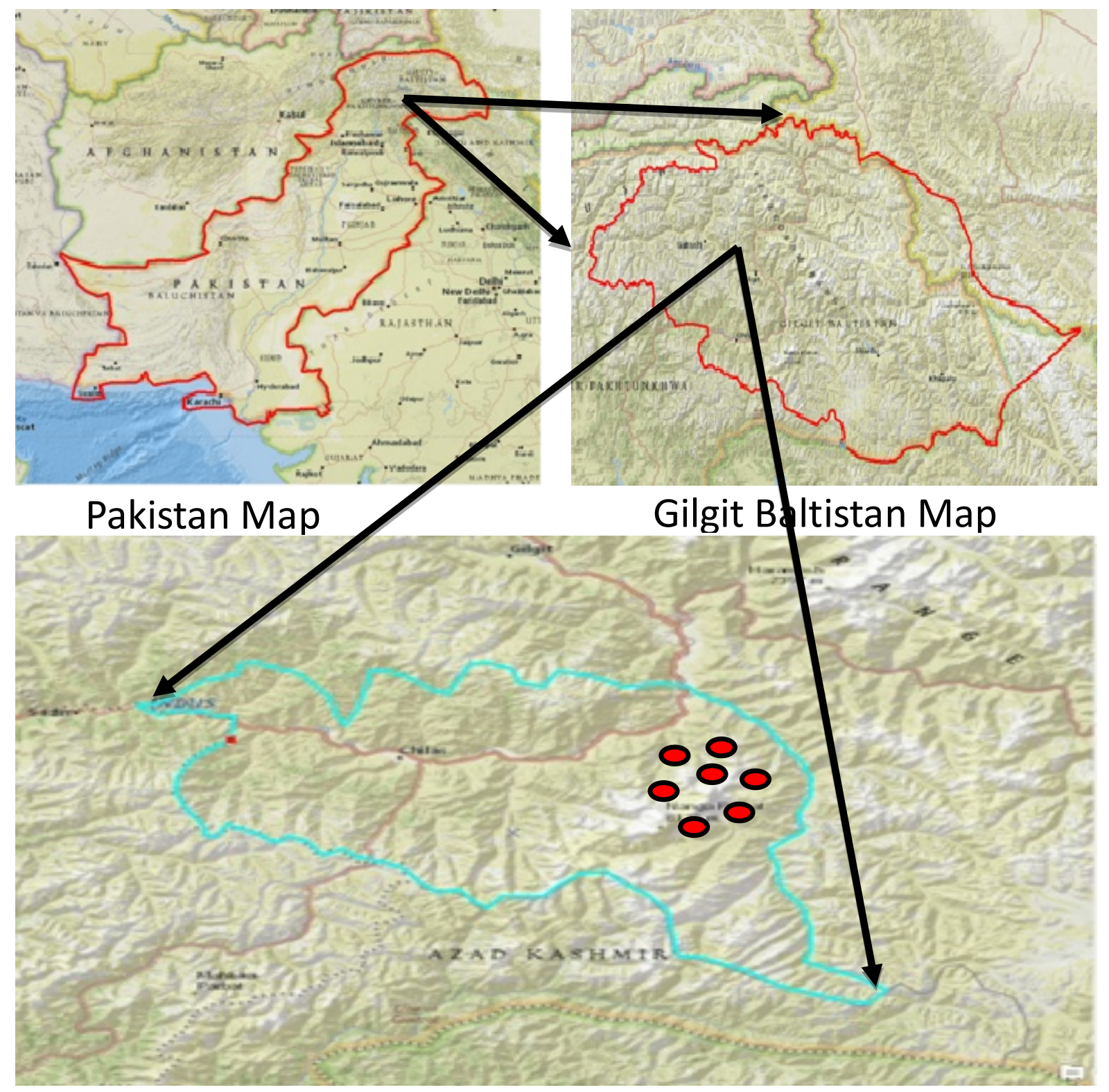

Diamir Map, (Study Site o Fairy Meadows)

Fig. 1. Map of the study area. (A)Pakistan (B) Gilgit Baltistan (C) Diamir

\section{Demographic Information}

A total of 146 informants of different age groups were interviewed, including 101 (69.16\%) men and 45 (30.82\%) women. Among these, 30 (20.55\%) informants were less than 20 years old, $23(15.75 \%)$ were between 20 to 30 years, $28(19.18 \%)$ were 31 to 40 years, $29(19.86 \%)$ were 41 to 50 years, 21 $(14.38 \%)$ were 51 to 60 years and $15(10.27 \%)$ were 
above 60 years old. Among all the informants the oldest was 83 years old and the youngest 15 years old. The majority of the informants 104 (71.92\%) were married, $25(17.12 \%)$ were unmarried, and 2 $(0.68 \%)$ were widowed. Out of 146 informants, 101 $(70.55 \%)$ were illiterate and the rest literate. It was observed that $97(66.44 \%)$ informants were farmers, followed by $3(2.05 \%)$ retired from government jobs, $33(22.6 \%)$ were shepherds and $15(8.9 \%)$ followed other occupations. A majority of 124 (84.93\%) of the informants were residing in villages, and only a few $(15,22.07 \%)$ in cities. One-hundred-and-nineteen $(81.5 \%)$ informants had been residing in the surveyed area for more than 15 years, and 27 $(18.5 \%)$ less than 15 years. The study area has six ethnic groups. Among these, Sheen were in majority 74 (50.68\%), followed by Youshkon 38 (26.03\%), Khelochay 19 (13.01\%), 12 (8.22\%) gujar, 2 (1.37\%) Dulasgar and $1(0.68 \%)$ Kashmiri (Table1). All the ethnic groups have their own languages. However, the common and dominant language is Shina $(100 \%)$.

\section{Ethnobotanical documentation}

Different methods including semi-structured interviews, questionnaires, participant observations and walk-in-the-woods were used for the documentation of ethnobotanical data. In addition, focus group discussions were arranged to gain further information on medicinal plants knowledge of the community and authenticity of the data collected through semi-structured interviews. Interviews were conducted after obtaining oral prior informed consent from the participants. All interviews were conducted in Shina.

\section{Plant collection and identification}

Interviews and discussions were followed by a voucher specimen collection with the help field assistants. Specimens were systematically tagged, air-dried, pressed and mounted on herbarium sheets, each one labelled and voucher number allotted (Table 2). The botanical identification of the specimens was done by Prof. Dr. Rahmatullah Qureshi, a taxonomist at Pir Mehar Ali Shah, Arid Agriculture University Rawalpindi (PMAS AAUR), Pakistan and authenticated with the help of flora of Pakistan (www.eflora.com). The plant nomenclature follows the International Plant Name Index (IPNI). The specimens were also photographed during collection in the field, shown in (Fig. 2) and allocated a voucher number (Fig. 3). The mounted specimens were deposited in the Herbarium of Department of Botany PMAS AAUR, Pakistan.
The data recorded in the field was entered in Microsoft Excel. Data was organized in a tabulated form for presentation. The graphical representation was based on numerical data extracted.

Table 1. Demographic characteristics of Fairy Meadow.

\begin{tabular}{|c|c|c|c|}
\hline $\begin{array}{c}\text { Demographic } \\
\text { feature }\end{array}$ & Factor & $\begin{array}{l}\text { Number of } \\
\text { informants }\end{array}$ & Percentage \\
\hline \multirow{2}{*}{$\begin{array}{l}\text { Gender of } \\
\text { informants }\end{array}$} & Male & 101 & 69.18 \\
\hline & Female & 45 & 30.82 \\
\hline \multirow{6}{*}{$\begin{array}{c}\text { Age of } \\
\text { informants }\end{array}$} & $\begin{array}{l}\text { Less than } \\
20\end{array}$ & 30 & 20.55 \\
\hline & 20 to 30 & 23 & 15.75 \\
\hline & 31 to 40 & 28 & 19.18 \\
\hline & 41 to 50 & 29 & 19.86 \\
\hline & 51 to 60 & 21 & 14.38 \\
\hline & Above 60 & 15 & 10.27 \\
\hline \multirow{3}{*}{ Marital status } & Married & 104 & 71.92 \\
\hline & Unmarried & 25 & 17.12 \\
\hline & Widow & 2 & 0.68 \\
\hline \multirow{5}{*}{$\begin{array}{l}\text { Education } \\
\text { level }\end{array}$} & Illiterate & 101 & 69.18 \\
\hline & $\begin{array}{l}\text { Elementary } \\
\text { School }\end{array}$ & 25 & 17.12 \\
\hline & $\begin{array}{l}\text { Secondary } \\
\text { School }\end{array}$ & 7 & 4.79 \\
\hline & College & 9 & 6.16 \\
\hline & University & 4 & 2.74 \\
\hline \multirow{4}{*}{$\begin{array}{l}\text { Employment } \\
\text { Status }\end{array}$} & Farmer & 97 & 66.44 \\
\hline & Retired & 3 & 2.05 \\
\hline & Shepherd & 33 & 22.6 \\
\hline & Others & 13 & 8.9 \\
\hline \multirow{2}{*}{ Residence } & Village & 124 & 84.93 \\
\hline & City & 22 & 15.07 \\
\hline \multirow{6}{*}{ Ethnic group } & Youshkon & 38 & 26.03 \\
\hline & Sheen & 74 & 50.68 \\
\hline & Gujar & 12 & 8.22 \\
\hline & Dulasgar & 2 & 1.37 \\
\hline & Khelochey & 19 & 13.01 \\
\hline & Kashmiri & 1 & 0.68 \\
\hline \multirow{2}{*}{ Experience } & Herbalists & 2 & 1.37 \\
\hline & $\begin{array}{l}\text { Local } \\
\text { people }\end{array}$ & 144 & 98.63 \\
\hline \multirow{2}{*}{$\begin{array}{c}\text { Duration of } \\
\text { residence in } \\
\text { the surveyed } \\
\text { area }\end{array}$} & $\begin{array}{l}\text { Less than } \\
15 \text { years }\end{array}$ & 27 & 18.5 \\
\hline & $\begin{array}{l}\text { More than } \\
15 \text { years }\end{array}$ & 119 & 81.5 \\
\hline
\end{tabular}

\section{Data management}




\section{Statistical analysis}

The collected ethnomedicinal data was quantitatively analyzed using indices of relative frequency citation (RFC), use value (UV) and Pearson correlation coefficient (PCC).

\section{Relative Frequency of Citation (RFC)}

This index showed the local importance of each species. It is calculated using formula given by (Tardío and Pardo-de-Santayana, 2008):

\section{$\mathrm{RFC}=\mathrm{FC} / \mathrm{N}$}

Where FC is the number of informants mentioning the use of the species and $\mathrm{N}$ is the total number of informants involved in the survey $(\mathrm{N}=146)$, without considering the use-categories (Vitalini et al., 2013).

\section{Use Value (UV)}

The Use Value (UV) shows the relative importance of plants known locally. It was calculated using the formula given by (Phillips et al., 1994):

\section{$\mathbf{U V}=\sum \mathrm{Ui} / \mathbf{N}$}

Where $\mathrm{Ui}$ is the total number of uses mentioned by each informant for a single species and $\mathrm{N}$ is the total number of informants interviewed for a given species.

\section{Pearson Correlation Coefficient (PCC)}

For the interpretation of collected data, Pearson Correlation Coefficient was used which is a good measure to numerically quantify the nature of the linear relationship between two variables. It is the ratio of the covariance between two variables to their standard deviations.

\section{Results and discussion}

\section{Ethnographic information}

Details on informant composition are given in Table 1. The primary local language spoken in the area is Shina $(100 \%)$ while the other locally known languages include Khilocha, Gujriya, Kashmiri and Dulasgariya. During the fieldwork it became apparent that the local people self-medicated in case of any disease. Only in case of failure of home remedies, they consulted herbalists, and $2(1.37 \%)$ of the total informants accessed modern health facilities (Ayyanar and Ignacimuthu, 2005).

\section{Medicinal plants diversity}

A total of 90 plant species belonging to 77 genera and 49 different families were reported in this study
(Table 2). The dominant plant families were Asteraceae represented by 9 plant species (10\%) followed by Polygonaceae and Rosaceae with 8 plant species each (8.88\%), Pinaceae and Scrophulariaceae with 4 species each $(4.44 \%)$, Apiaceae, Fabaceae and Ranunculaceae with 3 species each ( $3.33 \%$ ), while rest of the families were represented by less than 3 species each (Fig. 4). The large number of medicinal plants in Asteraceae in this study are in agreement with a previous study in a neighboring district Skardu (Bano et al., 2012), and coincide with the findings of Ayyanar and Ignacimuthu (2005) and Benítez et al. (2010). Eleven different use categories were mentioned by the informants. Among the use categories, medicinal use was highest (Fig.5). The dominant life form used was herbs $(60 \%)$, followed by trees $(26 \%)$, shrubs (13\%), and woody climbers (1\%) (Fig. 6). These results are in correspondence with a previous ethnobotanical study in India (Ayyanar and Ignacimuthu, 2005). Most of the ethnomedicinally important plants were wild collected $(81 \%)$, and the remaining $19 \%$ cultivated (Fig. 7 ). The majority of the plants were only used medicinally, but 28 species were used both medicinally and for other purposes in the study area (Fig. 8). The most used parts were leaf $(27 \%)$, followed by fruit $(19 \%)$, root $(16 \%)$, stem $(12 \%)$, bark $(8 \%)$, flower $(6 \%)$, whole plant $(5 \%)$, gum $(3 \%)$, seed $(2 \%)$, cone and latex were used rarely (1\%) (Fig. 9). These results correlate clearly with a previous study in Gilgit Baltistan (Bano et al., 2012). Various forms of herbal preparations were used in the study area, the most common preparation methods were powder $(27 \%)$, decoction $(16 \%)$, direct use $(13 \%)$, juice $(11 \%)$, extract and paste $(8 \%)$ each, infusion $(5 \%)$, poultice $(4 \%)$ saag and smoke/fragrance $(3 \%)$ each and ash $(2 \%)$ (Fig. 10). These results closely resemble with the studies conducted in different parts of the world where leaves stood out as the used part (Giday et al., 2009; Prabhu et al., 2014; Ullah et al., 2013; Yabesh et al., 2014). The reason behind the excessive medicinal uses of leaves is easy collection, as compared to other parts (Giday et al., 2009). In addition, leaves often carry a high variety of different metabolites (Ghorbani, 2005). The reported plant species were used as herbal medicines for the treatment of 55 different health disorders. Most common diseases treated are stomach problems, cough, asthma, and fever. Details are shown (Fig.11). The common route of administration was oral $(81.7 \%)$, followed by external $(8.7 \%)$, topical $(7.7 \%)$ and inhalation $(1.9 \%)$ (Fig. 12). These findings are contrary to the results of Wazir et al. (2004). These similarities and differences may correspond to the cultural similarities and differences leading to the plant part use or purpose of use difference. 
Table 2. Medicinal Plants with botanical name, voucher number, family, local name, habit, part used, medicinal uses, UV, FC, RFC, mode of utilization, administration route and comparison with previous ethnobotanical data.

\begin{tabular}{|c|c|c|c|c|c|c|c|c|c|c|c|}
\hline $\begin{array}{l}\text { Scientific name } \\
\text { Voucher number }\end{array}$ & Family & Local name & Habit & $\begin{array}{l}\text { Part } \\
\text { used }\end{array}$ & Medicinal uses & UV & FC & RFC & $\begin{array}{l}\text { Mode } \\
\text { of } \\
\text { use }\end{array}$ & Administration & $\begin{array}{l}\text { Comparison } \\
\text { with previous } \\
\text { ethnobotanica } \\
\text { studies }\end{array}$ \\
\hline $\begin{array}{l}\text { Allium cepa L. } \\
\text { RW-647 }\end{array}$ & Alliaceae & $\begin{array}{l}\text { Paloan/ } \\
\text { Kasho }\end{array}$ & $\mathrm{H}$ & L, St & $\begin{array}{l}\text { Diarrhea, increases } \\
\text { blood pressure, } \\
\text { spice, salad. }\end{array}$ & 0.8 & 25 & 0.17 & $\mathrm{~J}, \mathrm{Di}$ & Or & $\begin{array}{l}1 \bullet 2 \bullet 3 \bullet 4=5 \bullet 6 \bullet \\
7 \bullet 8 \bullet 9 \bullet 10 \bullet 11 \bullet \\
12 \bullet 13 \bullet 14 \bullet\end{array}$ \\
\hline $\begin{array}{l}\text { Allium gilgiticum } \\
\text { L. } \\
\text { RW-648 }\end{array}$ & Alliaceae & Khatpa & $\mathrm{H}$ & $\mathrm{S}$ & $\begin{array}{l}\text { Pain, headache, } \\
\text { vegetable, forage. }\end{array}$ & 0.66 & 39 & 0.26 & $\mathrm{Sa}$ & Or & $\begin{array}{l}1 \bullet 2 \bullet 3 \bullet 4 \bullet 5 \bullet 6 \bullet \\
7 \bullet 8 \bullet 9 \bullet 10 \bullet 11 \bullet \\
12 \bullet 13 \bullet 14 \bullet\end{array}$ \\
\hline $\begin{array}{l}\text { Pistacia khinjuk L. } \\
\text { RW-698 }\end{array}$ & Anacardiaceae & Khakao & $\mathrm{T}$ & $\mathrm{G}, \mathrm{F}$ & $\begin{array}{l}\text { Cough, asthma, gum } \\
\text { used to remove } \\
\text { phlem, gum smoke } \\
\text { eye problems, hair } \\
\text { removing and broken } \\
\text { pots, forage, Wood } \\
\text { sold as fuel wood. }\end{array}$ & 1.1 & 41 & 0.28 & $\mathrm{Po}, \mathrm{P}$ & Or & $\begin{array}{l}1 \bullet 2 \bullet 3 \square 4 \square 5 \bullet 6 \square \\
7 \square 8 \bullet 9 \bullet 10 \bullet 11 \square \\
12 \bullet 13 \square 14 \bullet\end{array}$ \\
\hline $\begin{array}{l}\text { Bunium persicum } \\
\text { (Boiss) B. } \\
\text { Fedtsch. } \\
\text { RW-660 }\end{array}$ & Apiaceae & Hayao & $\mathrm{H}$ & $\mathrm{F}$ & $\begin{array}{l}\text { Pneumonia, } \\
\text { digestive, Spice, } \\
\text { aromatic, appetite } \\
\text { stimulant, herbal tea, } \\
\text { cough, stomachic, } \\
\text { fever, headache, } \\
\text { forage, high market } \\
\text { price. }\end{array}$ & 3.5 & 90 & 0.61 & Po, Di & Or & $\begin{array}{l}1 \bullet 2 \square 3 \bullet 4 \bullet 5 \bullet 6 \bullet \\
7 \bullet 8 \bullet 9 \bullet 10 \bullet 11 \bullet \\
12 \bullet 13 \bullet 14 \bullet\end{array}$ \\
\hline $\begin{array}{l}\text { Heracleum } \\
\text { candicans Wall. } \\
\text { ex DC. } \\
\text { RW-680 }\end{array}$ & Apiaceae & Polia & $\mathrm{H}$ & $\mathrm{L}, \mathrm{R}$ & $\begin{array}{l}\text { Forage, backbone } \\
\text { ache, stomachic, } \\
\text { digestive, healing } \\
\text { purpose. }\end{array}$ & 1.8 & 35 & 0.23 & Di, Po & To/Or & $\begin{array}{l}1 \bullet 2 \bullet 3 \bullet 4 \bullet 5 \bullet 6 \bullet \\
7 \bullet 8 \bullet 9 \bullet 10 \bullet 11 \bullet \\
12 \bullet 13 \bullet 14 \bullet\end{array}$ \\
\hline $\begin{array}{l}\text { Pimpinella } \\
\text { diversifolia DC. } \\
\text {.RW-700 }\end{array}$ & Apiaceae & Choro & $\mathrm{H}$ & $\mathrm{R}$ & $\begin{array}{l}\text { Intestinal worms, } \\
\text { asthma, reduce } \\
\text { blood thickness, } \\
\text { stomachic, used to } \\
\text { enhance digestion, } \\
\text { forage. }\end{array}$ & 2.4 & 69 & 0.47 & Po & Or & $\begin{array}{l}1 \bullet 2 \bullet 3 \bullet 4 \bullet 5 \bullet 6 \bullet \\
7 \bullet 8 \bullet 9 \bullet 10=11 \bullet \\
12 \bullet 13 \bullet 14 \bullet\end{array}$ \\
\hline $\begin{array}{l}\text { Asparagus } \\
\text { officinalis L. } \\
\text { RW-653 }\end{array}$ & Asparagaceae & Sharkachey & $\mathrm{H}$ & St & $\begin{array}{l}\text { Anti-inflammatory, } \\
\text { diabetes, } \\
\text { antioxidant, }\end{array}$ & 0.42 & 33 & 0.22 & Di, po & Or & $\begin{array}{l}1 \bullet 2 \bullet 3 \bullet 4 \bullet 5 \bullet 6 \bullet \\
7 \bullet 8 \bullet 9 \bullet 10 \bullet 11 \bullet \\
12 \bullet 13 \bullet 14 \bullet\end{array}$ \\
\hline
\end{tabular}

Published: 14 November 2019

http://dx.doi.org/10.32859/era.18.35.1-30 


\begin{tabular}{|c|c|c|c|c|c|c|c|c|c|c|c|}
\hline & & & & & $\begin{array}{l}\text { digestive, anti- } \\
\text { cancer, maintains } \\
\text { blood glucose level, } \\
\text { forage. }\end{array}$ & & & & & & \\
\hline $\begin{array}{l}\text { Anacyclus } \\
\text { pyrethrum L. } \\
\text { RW-649 }\end{array}$ & Asteraceae & Peli ponar & $\mathrm{H}$ & Wp & $\begin{array}{l}\text { Sore throat, eye } \\
\text { wash, toothache and } \\
\text { forage. }\end{array}$ & 0.33 & 12 & 0.08 & $\begin{array}{l}\text { De, } \\
\text { Po }\end{array}$ & E/Or & $\begin{array}{l}1 \bullet 2 \bullet 3 \bullet 4 \bullet 5 \bullet 6 \bullet \\
7 \bullet 8 \bullet 9 \bullet 10 \bullet 11 \bullet \\
12 \bullet 13 \bullet 14 \bullet\end{array}$ \\
\hline $\begin{array}{l}\text { Anaphalis } \\
\text { nepalensis } \\
\text { (Spreng) Hand.- } \\
\text { Mazz. } \\
\text { RW-650 }\end{array}$ & Asteraceae & Cheki ponar & $\mathrm{H}$ & $\mathrm{F}$ & $\begin{array}{l}\text { Stomachic, aromatic, } \\
\text { forage. }\end{array}$ & 0.57 & 11 & 0.07 & $\begin{array}{l}\text { Fu, } \\
\text { Po }\end{array}$ & Or & $\begin{array}{l}1 \bullet 2 \backsim 3 \bullet 4 \bullet 5 \bullet 6 \bullet \\
7 \bullet 8 \square 9 \bullet 10 \bullet 11 \bullet \\
12 \bullet 13 \bullet 14 \bullet\end{array}$ \\
\hline $\begin{array}{l}\text { Artemisia glacialis } \\
\text { L. } \\
\text { RW-651 }\end{array}$ & Asteraceae & Nerlay zoon & Sh & $L$ & $\begin{array}{l}\text { Diarrhea, nausea, } \\
\text { intestinal worms, } \\
\text { helpful in pregnancy. }\end{array}$ & 1.66 & 32 & 0.21 & $\mathrm{~J}$ & Or & $\begin{array}{l}1 \bullet 2 \bullet 3 \bullet 4 \bullet 5 \bullet 6 \bullet \\
7 \bullet 8 \bullet 9 \bullet 10 \bullet 11 \bullet \\
12 \bullet 13 \bullet 14 \bullet\end{array}$ \\
\hline $\begin{array}{l}\text { Artemisia } \\
\text { maritima L. } \\
\text { RW-652 }\end{array}$ & Asteraceae & Zoon & Sh & $\mathrm{L}$ & $\begin{array}{l}\text { Diarrhea, nausea, } \\
\text { fever, Asthma, } \\
\text { headache, } \\
\text { stomachic, forage, } \\
\text { thatching. }\end{array}$ & 3.37 & 50 & 0.34 & $\mathrm{~J}$ & Or & $\begin{array}{l}1 \square 2 \bullet 3 \square 4 \square 5 \square 6 \bullet \\
7 \bullet 8 \square \\
9 \bullet 10 \bullet 11 \bullet 12 \square 13 \\
\bullet 14 \square\end{array}$ \\
\hline $\begin{array}{l}\text { Aster himalaicus } \\
\text { C. B. Clarke } \\
\text { RW-654 }\end{array}$ & Asteraceae & Zooti ponar & $\mathrm{H}$ & $\mathrm{F}$ & $\begin{array}{l}\text { Stomachic, aromatic, } \\
\text { forage. }\end{array}$ & 0.42 & 5 & 0.03 & $\begin{array}{l}\text { Fu, } \\
\text { Po }\end{array}$ & Or & $\begin{array}{l}1 \bullet 2 \bullet 3 \bullet 4 \bullet 5 \bullet 6 \bullet \\
7 \bullet 8 \bullet 9 \bullet 10 \bullet 11 \bullet \\
12 \bullet 13 \bullet 14 \bullet\end{array}$ \\
\hline $\begin{array}{l}\text { Cichorium intybus } \\
\text { L. } \\
\text { RW-665 }\end{array}$ & Asteraceae & Cheti char & $\mathrm{H}$ & Wp & $\begin{array}{l}\text { Healing of wounds, } \\
\text { remove dead cells } \\
\text { from wounds, bowls } \\
\text { paste is also applied } \\
\text { to cure boil, forage. }\end{array}$ & 1.14 & 29 & 0.19 & Ex & $E$ & $\begin{array}{l}1 \square 2 \bullet 3 \backsim 4 \square 5 \square 6 \square \\
7 \square 8 \bullet 9 \bullet 10 \bullet 11 \square \\
12 \bullet 13 \square 14 \bullet\end{array}$ \\
\hline $\begin{array}{l}\text { Echinops } \\
\text { echinatus Roxb. } \\
\text { RW-669 }\end{array}$ & Asteraceae & Janchey & $\mathrm{H}$ & $W p$ & $\begin{array}{l}\text { Stomachic, cough, } \\
\text { Arthritis, analgesic, } \\
\text { antibacterial, } \\
\text { antioxidant, diuretic, } \\
\text { forage. }\end{array}$ & 1 & 17 & 0.11 & $\begin{array}{l}\text { De, } \\
\text { Ex }\end{array}$ & Or & $\begin{array}{l}1 \square 2 \bullet 3 \bullet 4 \square 5 \bullet 6 \square \\
7 \square 8 \square 9 \bullet 10 \bullet 11 \bullet \\
12 \bullet 13 \bullet 14 \bullet\end{array}$ \\
\hline $\begin{array}{l}\text { Gnaphalium } \\
\text { sSpp. } \\
\text { RW-679 }\end{array}$ & Asteraceae & Unknown & $\mathrm{H}$ & $\mathrm{L}$ & $\begin{array}{l}\text { Constipation, } \\
\text { menses, sciatica, } \\
\text { forage. }\end{array}$ & 0.42 & 15 & 0.10 & $\mathrm{Di}$ & Or & $\begin{array}{l}1 \bullet 2 \bullet 3 \bullet 4 \bullet 5 \bullet 6 \bullet \\
7 \bullet 8 \bullet 9 \bullet 10 \bullet 11 \bullet \\
12 \bullet 13 \bullet 14 \bullet\end{array}$ \\
\hline $\begin{array}{l}\text { Saussurea } \\
\text { gossypiphora D. } \\
\text { Don } \\
\text { RW-721 } \\
\end{array}$ & Asteraceae & Bushi Ponar & $\mathrm{H}$ & $F$ & $\begin{array}{l}\text { Asthma, pneumonia, } \\
\text { stomach problem, } \\
\text { flue, headache, } \\
\text { improve circulation, }\end{array}$ & 4 & 77 & 0.52 & Po, In & Or & $\begin{array}{l}1 \bullet 2 \bullet 3 \bullet 4 \bullet 5 \bullet 6 \bullet \\
7 \bullet 8 \bullet 9 \bullet 10 \bullet 11 \bullet \\
12 \bullet 13 \bullet 14 \bullet\end{array}$ \\
\hline
\end{tabular}


Ethnobotany Research and Applications

\begin{tabular}{|c|c|c|c|c|c|c|c|c|c|c|c|}
\hline & & & & & $\begin{array}{l}\text { menstrual cycle, } \\
\text { cord umbilical cord. }\end{array}$ & & & & & & \\
\hline $\begin{array}{l}\text { Xanthium } \\
\text { strumarium L. } \\
\text { RW-733 }\end{array}$ & Asteraceae & $\begin{array}{l}\text { Dadi poshy } \\
\text { char }\end{array}$ & $\mathrm{H}$ & $\mathrm{L}, \mathrm{St}$ & Swelling, boils. & 0.87 & 15 & 0.10 & $P$ & To & $\begin{array}{l}1 \bullet 2 \bullet 3 \bullet 4 \bullet 5 \bullet 6 \bullet \\
7 \bullet 8 \bullet 9 \bullet 10 \bullet 11 \bullet \\
12 \bullet 13 \bullet 14 \bullet\end{array}$ \\
\hline $\begin{array}{l}\text { Berberis lycium } \\
\text { Royle } \\
\text { RW-656 }\end{array}$ & Berberidaceae & Chorko & Sh & Wp & $\begin{array}{l}\text { Bone fracture, } \\
\text { pneumonia, } \\
\text { headache, } \\
\text { stomachic, arthritis, } \\
\text { wound healing, } \\
\text { delivery, forage, } \\
\text { economic. }\end{array}$ & 5.47 & 141 & 0.96 & $\begin{array}{l}\mathrm{Po}, \\
\mathrm{Pa}, \\
\mathrm{De}\end{array}$ & To/Or & $\begin{array}{l}1 \square 2 \square 3 \square 4 \square 5 \square 6 \square \\
7 \square 8 \square 9 \square 10 \bullet 11 \square \\
12 \bullet 13 \bullet 14 \square\end{array}$ \\
\hline $\begin{array}{l}\text { Betula utilis D. } \\
\text { Don } \\
\text { RW-658 }\end{array}$ & Betulaceae & Jonji & $\mathrm{T}$ & $\mathrm{B}, \mathrm{St}$ & $\begin{array}{l}\text { Religious, spiritual, } \\
\text { bark smoke used for } \\
\text { removal of placenta } \\
\text { after delivery, } \\
\text { storage of } \\
\text { butter,Thatching, pot } \\
\text { making, wood and } \\
\text { bark sold in market } \\
\text { with high price }\end{array}$ & 1.87 & 108 & 0.73 & $\begin{array}{l}\text { De, } \\
\text { Fu }\end{array}$ & In & $\begin{array}{l}1 \bullet 2 \square 3 \square 4 \square 5 \bullet 6 \square \\
7 \bullet 8 \bullet 9 \bullet 10 \square 11 \square \\
12 \square 13 \bullet 14 \bullet\end{array}$ \\
\hline $\begin{array}{l}\text { Onosma hispida } \\
\text { Wall. ex G. Don. } \\
\text { RW-692 }\end{array}$ & Boraginaceae & Sharong & $\mathrm{H}$ & $\mathrm{R}, \mathrm{L}$ & $\begin{array}{l}\text { Throat infection, } \\
\text { menses, stomachic, } \\
\text { general pain in body, } \\
\text { asthma, hair and } \\
\text { face coloring, } \\
\text { lipstick, forage. }\end{array}$ & 1.75 & 35 & 0.23 & $\mathrm{De}$ & E/Or & $\begin{array}{l}1 \bullet 2 \bullet 3 \bullet 4-5 \bullet 6 \bullet \\
7 \bullet 8 \bullet 9 \bullet 10 \bullet 11 \bullet \\
12-13 \bullet 14 \bullet\end{array}$ \\
\hline $\begin{array}{l}\text { Capparis spinosa } \\
\text { Juss. } \\
\text { RW-661 }\end{array}$ & Capparidaceae & Loi margan & Sh & $\mathrm{F}, \mathrm{R}$ & $\begin{array}{l}\text { Sciatica, } \\
\text { rheumatism, } \\
\text { backache, diuretic, } \\
\text { anti-dandruff, } \\
\text { arthritis. }\end{array}$ & 0.42 & 17 & 0.11 & $\mathrm{Di}$ & Or & $\begin{array}{l}1 \bullet 2 \bullet 3 \square 4 \square 5 \square 6 \square \\
7 \bullet 8 \bullet 9 \square 10 \square 11 \square \\
12 \bullet 13 \bullet 14 \square\end{array}$ \\
\hline $\begin{array}{l}\text { Silene vulgaris } \\
\text { (Moench) Garcke } \\
\text { RW-723 }\end{array}$ & $\begin{array}{l}\text { Caryophyllace } \\
\text { ae }\end{array}$ & Unknown & $\mathrm{H}$ & $\mathrm{L}$ & $\begin{array}{l}\text { Cattle forage, } \\
\text { intestinal worms, } \\
\text { kidney problem. }\end{array}$ & 0.14 & 13 & 0.08 & $\mathrm{Di}$ & Or & $\begin{array}{l}1 \bullet 2 \bullet 3 \bullet 4 \bullet 5 \bullet 6 \bullet \\
7 \bullet 8 \bullet 9 \bullet 10 \bullet 11 \bullet \\
12 \bullet 13 \bullet 14 \bullet\end{array}$ \\
\hline $\begin{array}{l}\text { Chenopodium } \\
\text { album L. } \\
\text { RW-663 }\end{array}$ & $\begin{array}{l}\text { Chenopodiace } \\
\text { ae }\end{array}$ & Kunaw & $\mathrm{H}$ & $\mathrm{S}$ & Constipation, forage. & 0.37 & 9 & 0.06 & $\mathrm{Sa}$ & Or & $\begin{array}{l}1 \bullet 2 \bullet 3 \bullet 4 \square 5 \square 6 \square \\
7 \bullet 8 \bullet 9 \bullet 10 \bullet 11 \square \\
12 \bullet 13 \square 14 \square\end{array}$ \\
\hline
\end{tabular}


Ethnobotany Research and Applications

\begin{tabular}{|c|c|c|c|c|c|c|c|c|c|c|c|}
\hline $\begin{array}{l}\text { Hylotelephium } \\
\text { ewersi (Ledeb.) } \\
\text { H. Ohba } \\
\text { RW-722 }\end{array}$ & Crassulaceae & Teetar & $\mathrm{H}$ & Wp & $\begin{array}{l}\text { Constipation, } \\
\text { Stomachic. }\end{array}$ & 2 & 80 & 0.54 & Po & Or & $\begin{array}{l}1 \bullet 2 \bullet 3 \bullet 4 \bullet 5 \bullet 6 \bullet \\
7 \bullet 8 \bullet 9 \bullet 10 \bullet 11 \bullet \\
12 \bullet 13 \bullet 14 \bullet\end{array}$ \\
\hline $\begin{array}{l}\text { Juniperus } \\
\text { communis L. } \\
\text { RW-684 }\end{array}$ & Cupressaceae & Muthari & $\mathrm{T}$ & $\mathrm{F}, \mathrm{st}, \mathrm{L}$ & $\begin{array}{l}\text { Cold, fever, ash for } \\
\text { snuff, roots for } \\
\text { stitching of wooden } \\
\text { pots, bone fracture, } \\
\text { thatching. }\end{array}$ & 1.8 & 21 & 0.14 & $\begin{array}{l}\text { Po, } \\
\text { As }\end{array}$ & Or & $\begin{array}{l}1 \bullet 2 \backsim 3 \square 4 \square 5 \bullet 6 \bullet \\
7 \bullet 8 \bullet 9 \bullet 10 \square 11 \bullet \\
12 \bullet 13 \bullet 14 \square\end{array}$ \\
\hline $\begin{array}{l}\text { Juniperus excelsa } \\
\text { M. Bieb. } \\
\text { RW-685 }\end{array}$ & Cupressaceae & Chilli & $\mathrm{T}$ & $\begin{array}{l}\text { F,L, B, } \\
\text { St }\end{array}$ & $\begin{array}{l}\text { Berries for diabetes, } \\
\text { tuberculosis, Smoke } \\
\text { for bad evils, } \\
\text { rheumatism, burns, } \\
\text { cold fever, arthritis, } \\
\text { typhoid fever, wound } \\
\text { healing, leaves ash } \\
\text { snuff, kidney stone, } \\
\text { thatching, fuel, } \\
\text { furniture, berries } \\
\text { have high market } \\
\text { value. }\end{array}$ & 3.07 & 59 & 0.40 & $\begin{array}{l}\mathrm{Pa}, \\
\mathrm{Fu}\end{array}$ & To/Or & $\begin{array}{l}1 \square 2 \bullet 3 \square 4 \square 5 \bullet 6 \square \\
7 \bullet 8 \square 9 \bullet 10 \square 11 \square \\
12 \bullet 13 \square 14 \square\end{array}$ \\
\hline $\begin{array}{l}\text { Elaeagnus } \\
\text { angustifolia L. } \\
\text { RW-670 }\end{array}$ & Elaeagnaceae & Suzoon & $\mathrm{T}$ & $\mathrm{FI}, \mathrm{F}$ & $\begin{array}{l}\text { Cough, cold, } \\
\text { asthma, fever, } \\
\text { mental relaxation, } \\
\text { fragrant, joshanda. }\end{array}$ & 1.5 & 31 & 0.21 & $\mathrm{Di}$ & Or & $\begin{array}{l}1 \square 2 \bullet 3 \square 4 \square 5 \square 6 \square \\
7 \square 8 \square 9 \bullet 10 \square 11 \square \\
12 \bullet 13 \bullet 14 \square\end{array}$ \\
\hline $\begin{array}{l}\text { Hippophae } \\
\text { rhamnoides (L.) } \\
\text { A. Nelson } \\
\text { RW-681 }\end{array}$ & Elaeagnaceae & Buro & Sh & $\mathrm{F}, \mathrm{L}$ & $\begin{array}{l}\text { Anti-cancer, cough, } \\
\text { skin care, headache, } \\
\text { congestion of } \\
\text { bronchi, anti- } \\
\text { bacterial and viral, } \\
\text { antioxidant, tseeds } \\
\text { have high market } \\
\text { price, fence. }\end{array}$ & 5 & 65 & 0.44 & $\begin{array}{l}\mathrm{De} \\
\mathrm{Po} \\
\mathrm{In}, \mathrm{Pa}\end{array}$ & To/Or & $\begin{array}{l}1 \square 2 \square 3 \square 4 \square 5 \square 6 \square \\
7 \square 8 \square 9 \square 10 \square 11 \square \\
12 \square 13 \square 14 \square\end{array}$ \\
\hline $\begin{array}{l}\text { Ephedra } \\
\text { gerardiana Wall. } \\
\text { ex Stapf } \\
\text { RW-671 }\end{array}$ & Ephedraceae & Soontal & Sh & $\mathrm{S}$ & $\begin{array}{l}\text { Cigarette, cough, } \\
\text { cold, flue, nose } \\
\text { treatment and in } \\
\text { joshanda. }\end{array}$ & 0.28 & 27 & 0.18 & $\begin{array}{l}\mathrm{De}, \\
\mathrm{Po}\end{array}$ & Or & $\begin{array}{l}1 \square 2 \bullet 3 \bullet 4 \square 5 \square 6 \square \\
7 \square 8 \square 9 \square 10 \square 11 \square \\
12 \square 13 \square 14 \square\end{array}$ \\
\hline $\begin{array}{l}\text { Equisetum } \\
\text { hyemale L. } \\
\text { RW-672 }\end{array}$ & Equisitaceae & Cheyao & $\mathrm{H}$ & $\mathrm{S}$ & $\begin{array}{l}\text { Kidney problems, } \\
\text { skin and mouth } \\
\text { dryness, forage. }\end{array}$ & 0.87 & 37 & 0.25 & $\mathrm{~J}$ & Or & $\begin{array}{l}1 \bullet 2 \bullet 3 \bullet 4 \bullet 5 \bullet 6 \bullet \\
7 \bullet 8 \bullet 9 \bullet 10 \bullet 11 \bullet \\
12 \bullet 13 \bullet 14 \bullet\end{array}$ \\
\hline
\end{tabular}

Published: 14 November 2019

http://dx.doi.org/10.32859/era.18.35.1-30 
Ethnobotany Research and Applications

\begin{tabular}{|c|c|c|c|c|c|c|c|c|c|c|c|}
\hline $\begin{array}{l}\text { Astragalus } \\
\text { gilgitensis Ali } \\
\text { RW-655 }\end{array}$ & Fabaceae & Khukunay & $\mathrm{H}$ & $\mathrm{F}$ & Digestive, Forage. & 0.3 & 34 & 0.23 & $\mathrm{Di}$ & Or & $\begin{array}{l}1 \bullet 2 \bullet 3 \bullet 4 \bullet 5 \bullet 6 \bullet \\
7 \bullet 8 \bullet 9 \bullet 10 \bullet 11 \bullet \\
12 \bullet 13 \bullet 14 \bullet\end{array}$ \\
\hline $\begin{array}{l}\text { Cicer } \\
\text { microphyllum L. } \\
\text { RW-664 }\end{array}$ & Fabaceae & $\begin{array}{l}\text { Aseel } \\
\text { Khukunay }\end{array}$ & $\mathrm{H}$ & $\mathrm{F}$ & Forage. & 0.85 & 21 & 0.14 & $\mathrm{Di}$ & Or & $\begin{array}{l}1 \bullet 2 \bullet 3 \bullet 4 \bullet 5 \bullet 6 \bullet \\
7 \bullet 8 \bullet 9 \bullet 10 \bullet 11 \bullet \\
12 \bullet 13 \bullet 14 \bullet\end{array}$ \\
\hline $\begin{array}{l}\text { Medicago sativa } \\
\text { L. } \\
\text { RW-688 }\end{array}$ & Fabaceae & Rishka/lshpit & $\mathrm{H}$ & $\mathrm{S}$ & $\begin{array}{l}\text { lactogogue, saag, } \\
\text { fodder and forage. }\end{array}$ & 1 & 33 & 0.22 & $\mathrm{Sa}$ & Or & $\begin{array}{l}1 \bullet 2 \bullet 3 \square 4 \square 5 \bullet 6 \bullet \\
7 \bullet 8 \square 9 \bullet 10 \bullet 11 \square \\
12 \bullet 13 \bullet 14 \square\end{array}$ \\
\hline $\begin{array}{l}\text { Quercus incana } \\
\text { Bartra } \\
\text { mRW-709 }\end{array}$ & Fagaceae & Bani & $\mathrm{T}$ & St, L,R & $\begin{array}{l}\text { Furniture, cattle } \\
\text { forage, fuel wood, } \\
\text { thatching, wood is } \\
\text { sold in market as } \\
\text { fuel and timber } \\
\text { wood. }\end{array}$ & 0.14 & 35 & 0.23 & $\mathrm{Di}$ & Or & $\begin{array}{l}1 \bullet 2 \bullet 3 \bullet 4 \bullet 5 \bullet 6 \bullet \\
7 \bullet 8 \bullet \\
9 \bullet 10 \bullet 11 \bullet 12 \bullet 13 \\
\bullet 14 \bullet\end{array}$ \\
\hline $\begin{array}{l}\text { Gentiana kurroo } \\
\text { Royle } \\
\text { RW-677 }\end{array}$ & Gentianaceae & Cheti char & $\mathrm{H}$ & $\mathrm{S}, \mathrm{L}, \mathrm{F}$ & $\begin{array}{l}\text { Healing, removal of } \\
\text { dead cells, boils, } \\
\text { headache, cold, } \\
\text { fever, malaria, } \\
\text { forage. }\end{array}$ & 1.6 & 50 & 0.34 & $\mathrm{~Pa}, \mathrm{~J}$ & E/Or & $\begin{array}{l}1 \bullet 2 \bullet 3 \bullet 4 \bullet 5 \bullet 6 \bullet \\
7 \bullet 8 \bullet 9 \bullet 10 \bullet 11 \bullet \\
12 \bullet 13 \bullet 14 \bullet\end{array}$ \\
\hline $\begin{array}{l}\text { Swertia } \\
\text { marginata Royle. } \\
\text { RW-726 }\end{array}$ & Gentianaceae & Bangra & $\mathrm{H}$ & $\mathrm{L}$ & $\begin{array}{l}\text { Stomachic, Cancer, } \\
\text { fever, diuretic, } \\
\text { smoking, forage. }\end{array}$ & 0.42 & 10 & 0.06 & Po & Or & $\begin{array}{l}1 \bullet 2 \bullet 3 \bullet 4 \bullet 5 \bullet 6 \bullet \\
7 \bullet 8 \bullet 9 \bullet 10 \bullet 11 \bullet \\
12 \bullet 13 \bullet 14 \bullet\end{array}$ \\
\hline $\begin{array}{l}\text { Geranium } \\
\text { pratense L. } \\
\text { RW-678 }\end{array}$ & Geraniaceae & $\begin{array}{l}\text { Garoo/ratanj } \\
\text { ok }\end{array}$ & $\mathrm{H}$ & $\mathrm{R}$ & $\begin{array}{l}\text { After delivery to } \\
\text { reduce abdominal } \\
\text { pain, backache, } \\
\text { forage and milk } \\
\text { production in cattle. }\end{array}$ & 0.6 & 31 & 0.21 & Po & Or & $\begin{array}{l}1 \bullet 2 \bullet 3 \bullet 4 \square 5 \bullet 6 \bullet \\
7 \bullet 8 \bullet 9 \bullet 10 \bullet 11 \bullet \\
12 \bullet 13 \bullet 14 \bullet\end{array}$ \\
\hline $\begin{array}{l}\text { Ribes alpestre } \\
\text { Wall.ex. Decne. } \\
\text { RW-713 }\end{array}$ & $\begin{array}{l}\text { Grossulariacea } \\
\text { e }\end{array}$ & $\begin{array}{l}\text { Mayar } \\
\text { shatoo/Shat } \\
\text { oo }\end{array}$ & Sh & $\mathrm{F}$ & $\begin{array}{l}\text { Fever, burns, } \\
\text { blisters, coolant and } \\
\text { forage. }\end{array}$ & 0.37 & 23 & 0.15 & $\mathrm{~J}$ & Or & $\begin{array}{l}1 \bullet 2 \bullet 3 \bullet 4 \bullet 5 \bullet 6 \bullet \\
7 \bullet 8 \bullet 9 \bullet 10 \bullet 111 \\
12 \bullet 13 \bullet 14 \bullet\end{array}$ \\
\hline $\begin{array}{l}\text { Juglans regia L. } \\
\text { RW-683 }\end{array}$ & Juglandacae & Ashoey & $\mathrm{T}$ & $\mathrm{B}, \mathrm{L}, \mathrm{F}$ & $\begin{array}{l}\text { Cardiac, Brain tonic, } \\
\text { bark for maswak, } \\
\text { flesh of fresh fruit as } \\
\text { coloring agent, } \\
\text { leaves insect } \\
\text { repellent, cooking oil, } \\
\text { fruit have high } \\
\text { market value. }\end{array}$ & 2 & 61 & 0.41 & $\begin{array}{l}\mathrm{De} \\
\mathrm{Di}\end{array}$ & Or & $\begin{array}{l}1 \bullet 2 \bullet 3 \sqsubset 4 \bullet 5 \square 6 \square \\
7 \bullet 8 \bullet 9 \bullet 10 \square 11 \bullet \\
12 \bullet 13 \bullet 14 \bullet\end{array}$ \\
\hline
\end{tabular}




\begin{tabular}{|c|c|c|c|c|c|c|c|c|c|c|c|}
\hline $\begin{array}{l}\text { Mentha longifolia } \\
\text { (L.) L. } \\
\text { RW-689 }\end{array}$ & Labiateae & Phileel & $\mathrm{H}$ & $\mathrm{L}$ & $\begin{array}{l}\text { Tooth ache, nausea, } \\
\text { cough, fever, } \\
\text { diarrhea, intestinal } \\
\text { worms, blood } \\
\text { purifier, used for } \\
\text { blood reduction, } \\
\text { healing of wounds. }\end{array}$ & 3.71 & 64 & 0.43 & $\begin{array}{l}\mathrm{Pa}, \\
\mathrm{Di}, \mathrm{Po}\end{array}$ & Or & $\begin{array}{l}1 \square 2 \square 3 \bullet 4 \square 5 \square 6 \bullet \\
7 \bullet 8 \square 9 \bullet 10 \bullet 11 \bullet \\
12 \bullet 13 \bullet 14 \bullet\end{array}$ \\
\hline $\begin{array}{l}\text { Thymus linearis } \\
\text { L. } \\
\text { RW-727 }\end{array}$ & Labiateae & Tomurom & Sh & $\mathrm{L}$ & $\begin{array}{l}\text { Oxygenation, flue, } \\
\text { stomachic, cough, } \\
\text { headache, fever, } \\
\text { pneumonia, blood } \\
\text { purifier, against } \\
\text { obesity, weakness. }\end{array}$ & 5.06 & 130 & 0.89 & $\mathrm{De}$ & Or & $\begin{array}{l}1 \bullet 2 \square 3 \bullet 4 \square 5 \bullet 6 \bullet \\
7 \bullet 8 \bullet 9 \bullet 10 \bullet 11 \square \\
12 \bullet 13 \square 14 \bullet\end{array}$ \\
\hline $\begin{array}{l}\text { Isodon rugosus } \\
\text { (Wall. ex. Benth) } \\
\text { Codd) } \\
\text { RW-682 }\end{array}$ & Lamiaceae & Burdal & $\mathrm{H}$ & $\mathrm{L}$ & $\begin{array}{l}\text { Eye problem, } \\
\text { malaria, forage. }\end{array}$ & 1.11 & 31 & 0.21 & Ex & Or & $\begin{array}{l}1 \bullet 2 \bullet 3 \bullet 4 \bullet 5 \bullet 6 \bullet \\
7 \bullet 8 \bullet 9 \bullet 10 \bullet 11 \bullet \\
12 \bullet 13 \bullet 14 \bullet\end{array}$ \\
\hline $\begin{array}{l}\text { Ficus carica L. } \\
\text { RW- } 673\end{array}$ & Moraceae & $\begin{array}{l}\text { Angrezi } \\
\text { phang }\end{array}$ & $\mathrm{T}$ & F. La & $\begin{array}{l}\text { Kidney stone, blood } \\
\text { purifier, asthma, } \\
\text { obstruction in liver } \\
\text { and spleen, latex } \\
\text { used for blisters, } \\
\text { healing, fruit sold in } \\
\text { local market as a } \\
\text { asource of income. }\end{array}$ & 1.12 & 43 & 0.29 & $\mathrm{Di}$ & Or & $\begin{array}{l}1 \bullet 2 \bullet 3 \bullet 4 \backsim 5 \square 6 \square \\
7 \square 8 \square 9 \bullet 10 \backsim 11 \bullet \\
12 \bullet 13 \bullet 14 \bullet\end{array}$ \\
\hline $\begin{array}{l}\text { Ficus palmata L. } \\
\text { RW-674 }\end{array}$ & Moraceae & Phang & $\mathrm{T}$ & $\mathrm{F}, \mathrm{La}$ & $\begin{array}{l}\text { Asthma, Obstruction } \\
\text { in liver and spleen, } \\
\text { latex used for } \\
\text { blisters and healing }\end{array}$ & 1.3 & 40 & 0.27 & Di & Or & $\begin{array}{l}1 \bullet 2 \bullet 3 \bullet 4 \bullet 5 \bullet 6 \bullet \\
7 \bullet 8 \bullet 9 \bullet 10 \bullet 11 \bullet \\
12 \bullet 13 \bullet 14 \bullet\end{array}$ \\
\hline $\begin{array}{l}\text { Morus alba L. } \\
\text { RW-690 }\end{array}$ & Moraceae & $\begin{array}{l}\text { Peban } \\
\text { maroch/sho } \\
\text { maroch }\end{array}$ & $\mathrm{T}$ & $F$ & $\begin{array}{l}\text { Cough and facial } \\
\text { dropsy, throat } \\
\text { infection, memory } \\
\text { enhancer, jaundice, } \\
\text { fruit have high } \\
\text { market price. }\end{array}$ & 1.45 & 54 & 0.36 & $\mathrm{~J}, \mathrm{Po}$ & Or & $\begin{array}{l}1 \bullet 2 \bullet 3 \square 4 \square 5 \square 6 \square \\
7 \square 8 \bullet 9 \bullet 10 \backsim 11 \bullet \\
12 \bullet 13 \bullet 14 \bullet\end{array}$ \\
\hline $\begin{array}{l}\text { Morus nigra L. } \\
\text { RW-691 }\end{array}$ & Moraceae & $\begin{array}{l}\text { Kinay } \\
\text { maroch }\end{array}$ & $\mathrm{T}$ & $F$ & $\begin{array}{l}\text { Jaundice, sore } \\
\text { throat, cough, } \\
\text { fodder, economic, } \\
\text { fruit and wood have } \\
\text { market price, forage. }\end{array}$ & 0.57 & 25 & 0.17 & $\begin{array}{l}\mathrm{Di}, \mathrm{J} \\
\text { Po }\end{array}$ & Or & $\begin{array}{l}1 \bullet 2 \bullet 3 \bullet 4 \square 5 \square 6 \square \\
7 \square 8 \bullet 9 \bullet 10 \bullet 11 \bullet \\
12 \bullet 13 \bullet 14 \bullet\end{array}$ \\
\hline
\end{tabular}




\begin{tabular}{|c|c|c|c|c|c|c|c|c|c|c|c|}
\hline $\begin{array}{l}\text { Rhododendron } \\
\text { anthopogon D. } \\
\text { Don } \\
\text { RW-712 }\end{array}$ & Oleaceae & Susar & Sh & $\mathrm{L}$ & $\begin{array}{l}\text { Backbone ache, } \\
\text { better after delivery, } \\
\text { pneumonia, flue, } \\
\text { asthma, joint ache, } \\
\text { milk production and } \\
\text { blood purifier, } \\
\text { dizziness. }\end{array}$ & 5 & 109 & 0.74 & $\begin{array}{l}\mathrm{De} \\
\mathrm{Po}\end{array}$ & Or & $\begin{array}{l}1 \bullet 2 \bullet 3 \bullet 4 \bullet 5 \bullet 6 \bullet \\
7 \bullet 8 \bullet 9 \bullet 10 \bullet 11 \bullet \\
12 \bullet 13 \sqsubset 14 \bullet\end{array}$ \\
\hline $\begin{array}{l}\text { Pedicularis } \\
\text { pectinata Wall. ex } \\
\text { Benn. } \\
\text { RW-696 }\end{array}$ & $\begin{array}{l}\text { Orobanchacea } \\
\text { e }\end{array}$ & Unknown & $\mathrm{H}$ & $\mathrm{L}$ & $\begin{array}{l}\text { Stomachic, cardiac, } \\
\text { forage. }\end{array}$ & 0.12 & 31 & 0.21 & $\mathrm{Di}$ & Or & $\begin{array}{l}1 \bullet 2 \bullet 3 \bullet 4 \bullet 5 \bullet \\
6 \bullet 7 \bullet 8 \bullet 9 \bullet \\
10 \bullet 11 \bullet 12 \bullet \\
13 \bullet 14 \bullet\end{array}$ \\
\hline $\begin{array}{l}\text { Cedrus deodara } \\
\text { (Roxb. ex D. Don) } \\
\text { G. Don. } \\
\text { RW-662 }\end{array}$ & Pinaceae & Phuloosh & $\mathrm{T}$ & St, R, B & $\begin{array}{l}\text { Wood extract for } \\
\text { external parasites } \\
\text { and skin diseases of } \\
\text { goat, intestinal } \\
\text { worms, gum for } \\
\text { asthma, remove } \\
\text { pubic hair and } \\
\text { welding of broken } \\
\text { pots, wood is sold in } \\
\text { market as timber and } \\
\text { fuel wood with high } \\
\text { prices. }\end{array}$ & 1.44 & 40 & 0.27 & Ex & Or & $\begin{array}{l}1 \bullet 2 \bullet 3 \bullet 4 \bullet 5 \bullet 6 \\
7 \bullet 8 \bullet 9 \bullet 10 \bullet 11 \bullet \\
12 \bullet 13 \bullet 14 \bullet\end{array}$ \\
\hline $\begin{array}{l}\text { Picea smithiana } \\
\text { (Wall.) Boiss. } \\
\text { RW-699 }\end{array}$ & Pinaceae & Kachal & $\mathrm{T}$ & G & $\begin{array}{l}\text { Asthma, diarrhea, } \\
\text { tuberculosis, } \\
\text { diabetes, gum used } \\
\text { to repair broken } \\
\text { pots, hair removing. }\end{array}$ & 1.66 & 40 & 0.27 & $P$ & EOr & $\begin{array}{l}1 \bullet 2 \bullet 3 \bullet 4 \bullet 5 \bullet 6 \bullet \\
7 \bullet 8 \bullet 9 \bullet 10 \bullet 11 \bullet \\
12 \bullet 13 \bullet 14 \bullet\end{array}$ \\
\hline $\begin{array}{l}\text { Pinus gerardiana } \\
\text { Wall. ex D. Don } \\
\text { nRW-701 }\end{array}$ & Pinaceae & $\begin{array}{l}\text { Chalgoza/th } \\
\text { olesh }\end{array}$ & $\mathrm{T}$ & $F, G$, Co & $\begin{array}{l}\text { Fruit for headache, , } \\
\text { cone ash detergent, } \\
\text { fodder, fuel, } \\
\text { furniture, hair } \\
\text { removing, wood and } \\
\text { fruit have high } \\
\text { market value. }\end{array}$ & 1.33 & 63 & 0.43 & As, Di & Or/E & $\begin{array}{l}1 \bullet 2 \bullet 3 \bullet 4 \square 5 \bullet 6 \bullet \\
7 \bullet 8 \bullet 9 \bullet 10=11 \bullet \\
12 \bullet 13 \bullet 14 \bullet\end{array}$ \\
\hline $\begin{array}{l}\text { Pinus wallichiana } \\
\text { A. B. Jacks } \\
\text { RW-702 }\end{array}$ & Pinaceae & Chee & $\mathrm{T}$ & G & $\begin{array}{l}\text { Heart attack, gum } \\
\text { used to cure wound } \\
\text { in kidney, stomach } \\
\text { and urinary bladder, } \\
\text { bronchial } \\
\text { congestion, asthma, }\end{array}$ & 1.8 & 37 & 0.25 & $P$ & To/Or & $\begin{array}{l}1 \bullet 2 \bullet 3 \backsim 4 \bullet 5 \bullet 6 \bullet \\
7 \bullet 8 \bullet 9 \bullet 10 \square 11 \bullet \\
12 \bullet 13 \bullet 14 \bullet\end{array}$ \\
\hline
\end{tabular}

Published: 14 November 2019

http://dx.doi.org/10.32859/era.18.35.1-30 


\begin{tabular}{|c|c|c|c|c|c|c|c|c|c|c|c|}
\hline & & & & & $\begin{array}{l}\text { removal of pubic } \\
\text { hair, wood sold in } \\
\text { market as timber } \\
\text { wood. }\end{array}$ & & & & & & \\
\hline $\begin{array}{l}\text { Plantago } \\
\text { lanceolatae L. } \\
\text { RW-703 }\end{array}$ & $\begin{array}{l}\text { Plantaginacea } \\
\mathrm{e}\end{array}$ & $\begin{array}{l}\text { khapoy } \\
\text { patey }\end{array}$ & $\mathrm{H}$ & $\mathrm{L}$ & $\begin{array}{l}\text { Stops bleeding from } \\
\text { nose, animal graze. }\end{array}$ & 1.6 & 15 & 0.10 & $\mathrm{~Pa}$ & Or & $\begin{array}{l}1 \bullet 2 \bullet 3 \bullet 4 \bullet 5 \bullet \\
6 \bullet 7 \bullet 8 \bullet 9 \bullet \\
10 \bullet 11 \bullet 12 \bullet 13 \square \\
14 \bullet\end{array}$ \\
\hline $\begin{array}{l}\text { Limonium } \\
\text { cabulicum } \\
\text { (Boiss.) Kuntze } \\
\text { RW-686 }\end{array}$ & $\begin{array}{l}\text { Plumbaginace } \\
\text { ae }\end{array}$ & $\begin{array}{l}\text { Hazrat } \\
\text { Daroo }\end{array}$ & $\mathrm{H}$ & Wp & $\begin{array}{l}\text { Asthma, pneumonia, } \\
\text { tuberculosis, } \\
\text { bleeding from gums, } \\
\text { piles, thought to be } \\
\text { used for thousand } \\
\text { diseases, forage. }\end{array}$ & 2.66 & 47 & 0.32 & Po & Or & $\begin{array}{l}1 \bullet 2 \bullet 3 \bullet 4 \bullet 5 \bullet 6 \bullet \\
7 \bullet 8 \bullet 9 \bullet 10 \bullet 11 \bullet \\
12 \bullet 13 \bullet 14 \bullet\end{array}$ \\
\hline $\begin{array}{l}\text { Zea mays L. } \\
\text { RW-734 }\end{array}$ & Poaceae & Makaey & $\mathrm{H}$ & $\mathrm{F}$ & $\begin{array}{l}\text { Paste applied on } \\
\text { swelling parts, } \\
\text { blisters, as food, } \\
\text { fodder, high market } \\
\text { price. }\end{array}$ & 1.37 & 39 & 0.26 & $\mathrm{Po} / \mathrm{Pa}$ & Or & $\begin{array}{l}1 \bullet 2 \bullet 3 \bullet 4 \bullet 5 \bullet 6 \bullet \\
7 \bullet 8 \bullet \\
9 \bullet 10 \bullet 11 \bullet 12 \bullet 13 \\
\bullet 14 \bullet\end{array}$ \\
\hline $\begin{array}{l}\text { Bistorta } \\
\text { amplexicaulis (D. } \\
\text { Don) Greene } \\
\text { RW-697 }\end{array}$ & Polygonaceae & Shey lamay & $\mathrm{H}$ & $\mathrm{R}$ & $\begin{array}{l}\text { Backache, } \\
\text { stomachic, } \\
\text { lactogogue in cattle, } \\
\text { thatching, forage. }\end{array}$ & 1.5 & 56 & 0.38 & Po & Or & $\begin{array}{l}1 \bullet 2 \bullet 3 \bullet 4 \bullet 5 \bullet 6 \bullet \\
7 \bullet 8 \bullet 9 \bullet 10 \bullet 11 \bullet \\
12 \bullet 13 \bullet 14 \bullet\end{array}$ \\
\hline $\begin{array}{l}\text { Bistorta vivipara } \\
\text { L. } \\
\text { RW-659 }\end{array}$ & Polygonaceae & Chenga & $\mathrm{H}$ & $\mathrm{F}, \mathrm{R}$ & $\begin{array}{l}\text { Fruit edible, throat } \\
\text { infection, root for } \\
\text { piles, forage. }\end{array}$ & 0.71 & 29 & 0.19 & Di, Ex & Or & $\begin{array}{l}1 \bullet 2 \bullet 3 \bullet 4 \bullet 5 \bullet 6 \bullet \\
7 \bullet 8 \bullet 9 \bullet 10 \bullet 11 \bullet \\
12 \bullet 13 \bullet 14 \bullet\end{array}$ \\
\hline $\begin{array}{l}\text { Oxyria digyna (L.) } \\
\text { Hill } \\
\text { RW-693 }\end{array}$ & Polygonaceae & $\begin{array}{l}\text { Nerlay } \\
\text { Churki }\end{array}$ & $\mathrm{H}$ & L & $\begin{array}{l}\text { Cattle fodder, used } \\
\text { for better digestion. }\end{array}$ & 0.22 & 21 & 0.14 & $\overline{\mathrm{Di}}$ & Or & $\begin{array}{l}1 \bullet 2 \bullet 3 \bullet 4 \square 5 \bullet 6 \bullet \\
7 \bullet 8 \bullet 9 \square 10 \bullet 11 \bullet \\
12 \bullet 13 \bullet 14 \bullet\end{array}$ \\
\hline $\begin{array}{l}\text { Persicaria affinis } \\
\text { D. Don } \\
\text { RW-704 }\end{array}$ & Polygonaceae & $\begin{array}{l}\text { Shoni } \\
\text { chenga }\end{array}$ & $\mathrm{H}$ & $\mathrm{R}$ & $\begin{array}{l}\text { Fever, forage, } \\
\text { thatching. }\end{array}$ & 0.28 & 16 & 0.10 & Ex & Or & $\begin{array}{l}1 \bullet 2 \bullet 3 \bullet 4 \backsim 5 \bullet \\
6 \bullet 7 \bullet 8 \bullet 9 \bullet \\
10 \bullet 11 \bullet 12 \bullet 13 \bullet \\
14 \bullet\end{array}$ \\
\hline $\begin{array}{l}\text { Rheum australe } \\
\text { D. Don } \\
\text { RW- } 710\end{array}$ & Polygonaceae & $\begin{array}{l}\text { Margosh } \\
\text { chontal }\end{array}$ & $\mathrm{H}$ & $\mathrm{R}$ & $\begin{array}{l}\text { Intestinal worms } \\
\text { both human and } \\
\text { cattle, constipation, } \\
\text { hair and cloth } \\
\text { coloring, forage. }\end{array}$ & 1.5 & 60 & 0.41 & $\begin{array}{l}\text { Po, } \\
\text { De }\end{array}$ & Or/E & $\begin{array}{l}1 \bullet 2 \bullet 3 \bullet 4 \bullet 5 \bullet 6 \bullet \\
7 \bullet 8 \bullet 9 \bullet 10 \bullet 11 \bullet \\
12 \bullet 13 \bullet 14 \bullet\end{array}$ \\
\hline $\begin{array}{l}\text { Rheum } \\
\text { webbianum Wall. } \\
\text { RW-711 }\end{array}$ & Polygonaceae & Chontal & $\mathrm{H}$ & $\mathrm{R}$ & $\begin{array}{l}\text { Hair coloring, blood } \\
\text { purifier, stomachic, }\end{array}$ & 0.87 & 17 & 0.11 & Po, J & To/Or & $\begin{array}{l}1 \bullet 2 \bullet 3 \bullet 4 \bullet 5 \bullet 6 \bullet \\
7 \bullet 8 \square 9 \bullet 10 \bullet 11 \bullet \\
12 \bullet 13 \bullet 14 \bullet\end{array}$ \\
\hline
\end{tabular}

Published: 14 November 2019

http://dx.doi.org/10.32859/era.18.35.1-30 


\begin{tabular}{|c|c|c|c|c|c|c|c|c|c|c|c|}
\hline & & & & & $\begin{array}{l}\text { dyspepsia, tonic for } \\
\text { goat, sheep, forage. }\end{array}$ & & & & & & \\
\hline $\begin{array}{l}\text { Rumex hastatus } \\
\text { D. Don } \\
\text { RW-716 }\end{array}$ & Polygonaceae & Chorki & $\mathrm{H}$ & $W p$ & $\begin{array}{l}\text { Forage, anti-poison, } \\
\text { used for better } \\
\text { digestion, anti- } \\
\text { rusting. }\end{array}$ & 0.90 & 29 & 0.19 & $\mathrm{~J}$ & Or & $\begin{array}{l}1 \backsim 2 \bullet 3 \bullet 4 \square 5 \bullet 6 \bullet \\
7 \bullet 8 \bullet 9 \bullet 10 \bullet 11 \bullet \\
12 \bullet 13 \bullet 14 \bullet\end{array}$ \\
\hline $\begin{array}{l}\text { Rumex } \\
\text { nepalensis L. } \\
\text { RW-717 }\end{array}$ & Polygonaceae & Hubabol & $\mathrm{H}$ & $\mathrm{R}$ & $\begin{array}{l}\text { Healing, respiratory } \\
\text { disorders, extract } \\
\text { used for bile } \\
\text { reduction, remove } \\
\text { worms from } \\
\text { wounded sites, } \\
\text { cough. }\end{array}$ & 2.28 & 47 & 0.32 & $\begin{array}{l}\text { Po, } \\
\text { De }\end{array}$ & To/Or & 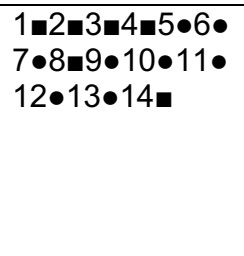 \\
\hline $\begin{array}{l}\text { Primula } \\
\text { macrophylla D. } \\
\text { Don } \\
\text { RW-705 }\end{array}$ & Primulaceae & Mabera & $\mathrm{H}$ & $\mathrm{L}$ & $\begin{array}{l}\text { Eye problem, } \\
\text { tobacco, forage. }\end{array}$ & 1.5 & 60 & 0.41 & Po & Or & $\begin{array}{l}1 \bullet 2 \bullet 3 \bullet 4 \square 5 \bullet 6 \bullet \\
7 \bullet 8 \bullet 9 \bullet 10 \bullet 11 \square \\
12 \bullet 13 \bullet 14 \bullet\end{array}$ \\
\hline $\begin{array}{l}\text { Adiantum } \\
\text { raddianum C. } \\
\text { Presl } \\
\text { IRW-646 }\end{array}$ & Pteriudaceae & Simbul Char & $\mathrm{H}$ & $\mathrm{L}$ & $\begin{array}{l}\text { Fever, diarrhea, } \\
\text { forage. }\end{array}$ & 1.25 & 30 & 0.20 & Ex & Or & $\begin{array}{l}1 \bullet 2 \bullet 3 \bullet 4 \bullet 5 \bullet 6 \bullet \\
7 \bullet 8 \bullet 9 \bullet 10 \bullet 11 \bullet \\
12 \bullet 13 \bullet 14 \bullet\end{array}$ \\
\hline $\begin{array}{l}\text { Punica granatum } \\
\text { L. } \\
\text { RW-708 }\end{array}$ & Punicaceae & Danoi & $\mathrm{T}$ & $\mathrm{F}$ & $\begin{array}{l}\text { Exocarp for kidney } \\
\text { stone, urinary } \\
\text { infection, intestinal } \\
\text { worms, cough, } \\
\text { edible fruit, } \\
\text { increases blood, fruit } \\
\text { have high market } \\
\text { value. }\end{array}$ & 1.30 & 49 & 0.33 & Po, di & Or & $\begin{array}{l}1 \bullet 2 \bullet 3 \bullet 4 \square 5 \square 6 \square \\
7 \bullet 8 \bullet 9 \square 10 \square 11 \square \\
12 \bullet 13 \bullet 14 \bullet\end{array}$ \\
\hline $\begin{array}{l}\text { Aconitum } \\
\text { heterophyllum } \\
\text { Wall. ex Royle } \\
\text { RW-645 }\end{array}$ & $\begin{array}{l}\text { Ranunculacea } \\
\text { e }\end{array}$ & Patrees & $\mathrm{H}$ & $\mathrm{R}, \mathrm{F}$ & $\begin{array}{l}\text { Reduce bile, } \\
\text { headache, cold } \\
\text { fever, cleans } \\
\text { digestive tract, } \\
\text { forage, sold in } \\
\text { market with high } \\
\text { price. }\end{array}$ & 3.11 & 79 & 0.54 & Po & Or & $\begin{array}{l}1 \bullet 2 \bullet 3 \bullet 4 \bullet 5 \bullet 6 \bullet \\
7 \bullet 8 \bullet \\
9 \bullet 10 \bullet 11 \bullet 12 \bullet 13 \\
\bullet 14 \bullet\end{array}$ \\
\hline $\begin{array}{l}\text { Delphinium } \\
\text { brunonianum } \\
\text { Royle } \\
\text { RW-668 }\end{array}$ & $\begin{array}{l}\text { Ranunculacea } \\
\text { e }\end{array}$ & $\begin{array}{l}\text { Saboonay } \\
\text { tokay }\end{array}$ & $\mathrm{H}$ & $\mathrm{F}$ & $\begin{array}{l}\text { Coolant, arthritis, } \\
\text { asthma, backbone } \\
\text { ache, forage. }\end{array}$ & 0.2 & 7 & 0.04 & $\mathrm{De}$ & Or & $\begin{array}{l}1 \bullet 2 \bullet 3 \bullet 4 \square 5 \bullet 6 \bullet \\
7 \bullet 8 \bullet 9 \backsim 10 \bullet 11 \bullet \\
12 \bullet 13 \bullet 14 \bullet\end{array}$ \\
\hline
\end{tabular}


Ethnobotany Research and Applications

\begin{tabular}{|c|c|c|c|c|c|c|c|c|c|c|c|}
\hline $\begin{array}{l}\text { Salix tetrasperma } \\
\text { Roxb. } \\
\text { RW-720 }\end{array}$ & $\begin{array}{l}\text { Ranunculacea } \\
\text { e }\end{array}$ & Bayo & $T$ & Wp & $\begin{array}{l}\text { Anti-diarrheal, } \\
\text { reduce tonsils, } \\
\text { forage, maswak. }\end{array}$ & 0.71 & 27 & 0.18 & $\mathrm{De}$ & Or & $\begin{array}{l}1 \backsim 2 \boxminus 3 \bullet 4 \bullet 5 \bullet 6 \bullet \\
7 \bullet 8 \bullet 9 \bullet 10 \bullet 11 \bullet \\
12 \bullet 13 \bullet 14 \bullet\end{array}$ \\
\hline $\begin{array}{l}\text { Fragaria nubicola } \\
\text { (Lindl. Ex Hoof.f.) } \\
\text { Lacaita } \\
\text { RW-676 }\end{array}$ & Rosaceae & Bursay & $\mathrm{H}$ & $\mathrm{F}$ & Wild fruit, forage & 0.22 & 20 & 0.13 & Di & Or & $\begin{array}{l}1 \bullet 2 \boxminus 3 \bullet 4 \bullet 5 \bullet 6 \bullet \\
7 \bullet 8 \bullet 9 \bullet 10 \bullet 11 \bullet \\
12 \bullet 13 \bullet 14 \bullet\end{array}$ \\
\hline $\begin{array}{l}\text { Malus domestica } \\
\text { Borkh. } \\
\text { RW-687 }\end{array}$ & Rosaceae & Paloi & $\mathrm{T}$ & $\mathrm{F}$ & $\begin{array}{l}\text { Blood purification, } \\
\text { Edible, health tonic, } \\
\text { fruit is being sold as } \\
\text { source of income in } \\
\text { market. }\end{array}$ & 1.12 & 33 & 0.22 & $\mathrm{Di}$ & Or & $\begin{array}{l}1 \bullet 2 \bullet 3 \bullet 4 \bullet 5 \bullet 6 \bullet \\
7 \bullet 8 \bullet 9 \bullet 10 \bullet 11 \bullet \\
12 \bullet 13 \bullet 14 \bullet\end{array}$ \\
\hline $\begin{array}{l}\text { Prunus } \\
\text { amygdalus L. } \\
\text { RW-706 }\end{array}$ & Rosaceae & Badam & $\mathrm{T}$ & $\mathrm{S}$ & $\begin{array}{l}\text { Kushta jaat, } \\
\text { increases blood, } \\
\text { eyesight, skin care, } \\
\text { lactogogue, brain } \\
\text { tonic, energizer, fruit } \\
\text { have high market } \\
\text { value. }\end{array}$ & 1.21 & 39 & 0.26 & $\mathrm{Di}, \mathrm{Po}$ & Or & $\begin{array}{l}1 \bullet 2 \bullet 3 \bullet 4 \bullet 5 \bullet 6 \bullet \\
7 \bullet 8 \bullet 9 \bullet 10 \square 11 \bullet \\
12 \bullet 13 \bullet 14 \bullet\end{array}$ \\
\hline $\begin{array}{l}\text { Prunus } \\
\text { armeniaca L. } \\
\text { RW-707 }\end{array}$ & Rosaceae & Joi/Jein & $\mathrm{T}$ & $\mathrm{F}, \mathrm{S}$ & $\begin{array}{l}\text { Diarrhea, cough, } \\
\text { edible, fruit have } \\
\text { high market value. }\end{array}$ & 0.9 & 36 & 0.24 & $\mathrm{Di}, \mathrm{J}$ & Or & $\begin{array}{l}1 \bullet 2 \bullet 3 \bullet 4 \square 5 \square 6 \bullet \\
7 \square 8 \bullet 9 \square 10 \square 11 \bullet \\
12 \bullet 13 \bullet 14 \bullet\end{array}$ \\
\hline $\begin{array}{l}\text { Prunus persica L. } \\
\text { RW-725 }\end{array}$ & Rosaceae & Aaro & $\mathrm{T}$ & $\mathrm{S}$ & $\begin{array}{l}\text { Seed coat used for } \\
\text { eye problem, fruit } \\
\text { edible }\end{array}$ & 0.33 & 16 & 0.10 & SC & $E$ & $\begin{array}{l}1 \bullet 2 \bullet 3 \bullet 4 \bullet 5 \bullet 6 \bullet \\
7 \bullet 8 \bullet 9 \bullet 10 \bullet 11 \bullet \\
12 \bullet 13 \bullet 14 \bullet\end{array}$ \\
\hline $\begin{array}{l}\text { Rosa webbiana } \\
\text { Wall. } \\
\text { RW-714 }\end{array}$ & Rosaceae & Shengai & Sh & $R, B, F$ & $\begin{array}{l}\text { Reduce blood } \\
\text { pressure, decrease } \\
\text { extra blood, tea, } \\
\text { edible fruit, forage, } \\
\text { dying cloth. }\end{array}$ & 1 & 46 & 0.31 & $\mathrm{De}$ & Or & $\begin{array}{l}1 \bullet 2 \bullet 3 \backsim 4 \square 5 \bullet 6 \bullet \\
7 \bullet 8 \bullet \\
9 \square 10 \bullet 11 \square 12 \bullet 13 \\
\bullet 14 \bullet\end{array}$ \\
\hline $\begin{array}{l}\text { Rubus ulmifolius } \\
\text { Schott } \\
\text { RW-715 }\end{array}$ & Rosaceae & Ounchi & $\mathrm{H}$ & $\mathrm{F}$ & Hepatitis, Edible. & 0.42 & 10 & 0.06 & $\mathrm{Di}$ & Or & $\begin{array}{l}1 \bullet 2 \bullet 3 \bullet 4 \bullet 5 \bullet \\
6 \bullet 7 \bullet 8 \bullet 9 \bullet \\
10 \bullet 11 \bullet 12 \bullet \\
13 \bullet 14 \bullet\end{array}$ \\
\hline $\begin{array}{l}\text { Spiraea canesens } \\
\text { D. Don } \\
\text { RW-724 }\end{array}$ & Rosaceae & Darai & Sh & $B$ & $\begin{array}{l}\text { Skin care, delivery, } \\
\text { forage, making } \\
\text { sticks. }\end{array}$ & 0.6 & 31 & 0.21 & $\mathrm{P}$ & $E$ & $\begin{array}{l}1 \bullet 2 \bullet 3 \bullet 4 \square 5 \bullet 6 \bullet \\
7 \\
\bullet 8 \bullet 9 \bullet 10 \square 11 \bullet 1 \\
2 \bullet 13 \bullet 14 \bullet\end{array}$ \\
\hline $\begin{array}{l}\text { Salix acmophylla } \\
\text { Boiss. } \\
\text { RW-718 }\end{array}$ & Salicaceae & Biyao & $\mathrm{T}$ & $\mathrm{L}$ & $\begin{array}{l}\text { Diarrhea, sweet latex } \\
\text { on leavereduce extra }\end{array}$ & 0.44 & 17 & 0.11 & Ex & Or & $\begin{array}{l}1 \bullet 2 \bullet 3 \bullet 45 \bullet 5 \bullet 6 \\
\bullet 7 \bullet 8 \bullet 9 \bullet 10-11 \\
\bullet 12 \bullet 13 \bullet 14 \bullet\end{array}$ \\
\hline
\end{tabular}

Published: 14 November 2019

http://dx.doi.org/10.32859/era.18.35.1-30 


\begin{tabular}{|c|c|c|c|c|c|c|c|c|c|c|c|}
\hline & & & & & $\begin{array}{l}\text { blood, pot making, } \\
\text { thatching. }\end{array}$ & & & & & & \\
\hline $\begin{array}{l}\text { Salix alba L. } \\
\text { RW-719 }\end{array}$ & Salicaceae & $\begin{array}{l}\text { Machor/mori } \\
\text { bayao }\end{array}$ & $T$ & L, St & $\begin{array}{l}\text { Leaves use as } \\
\text { forage, persistent } \\
\text { fever, anti-diarrheal, } \\
\text { pot making. }\end{array}$ & 1 & 39 & 0.26 & $\mathrm{~J}$ & Or & $\begin{array}{l}1 \square 2 \square 3 \bullet 4 \backsim 5 \bullet 6 \bullet \\
7 \bullet 8 \square 9 \bullet 10 \bullet 11 \bullet \\
12 \bullet 13 \bullet 14 \bullet\end{array}$ \\
\hline $\begin{array}{l}\text { Bergenia } \\
\text { stracheyi (Hook.f. } \\
\text { \& Thomson) Engl. } \\
\text { RW-657 }\end{array}$ & Saxifragaceae & koret/Sabsar & $\mathrm{H}$ & $\mathrm{R}$ & $\begin{array}{l}\text { Tuberculosis, } \\
\text { backache, healing, } \\
\text { reduce infertility, } \\
\text { stomachic, diarrhea, } \\
\text { burns, arthritis, } \\
\text { inflammation. }\end{array}$ & 3.71 & 75 & 0.51 & Po & E/Or & $\begin{array}{l}1 \bullet 2 \square 3 \backsim 4 \backsim 5 \bullet 6 \bullet \\
7 \bullet 8 \square 9 \bullet 10 \bullet 11 \bullet \\
12 \bullet 13 \square 14 \bullet\end{array}$ \\
\hline $\begin{array}{l}\text { Pedicularis } \\
\text { bicornuta L. } \\
\text { RW-694 }\end{array}$ & $\begin{array}{l}\text { Scrophulariace } \\
\text { ae }\end{array}$ & Unknown & $\mathrm{H}$ & L & $\begin{array}{l}\text { General body pain, } \\
\text { stomachic, sedative, } \\
\text { forage. }\end{array}$ & 0.4 & 7 & 0.04 & $\mathrm{De}$ & Or & $\begin{array}{l}1 \bullet 2 \bullet 3 \bullet 4 \bullet 5 \bullet 6 \bullet \\
7 \bullet 8 \bullet 9 \bullet 10 \bullet 11 \bullet \\
12 \bullet 13 \bullet 14 \bullet\end{array}$ \\
\hline $\begin{array}{l}\text { Pedicularis flava } \\
\text { Pall. } \\
\text { RW-695 }\end{array}$ & $\begin{array}{l}\text { Scrophulariace } \\
\text { ae }\end{array}$ & Qaziban & $\mathrm{H}$ & $\mathrm{L}$ & Asthma, forage. & 0.37 & 20 & 0.13 & $\mathrm{De}$ & Or & $\begin{array}{l}1 \bullet 2 \bullet 3 \bullet 4 \bullet 5 \bullet 6 \bullet \\
7 \bullet 8 \bullet 9 \bullet 10 \bullet 11 \bullet \\
12 \bullet 13 \bullet 14 \bullet\end{array}$ \\
\hline $\begin{array}{l}\text { Verbascum } \\
\text { thapsus L. } \\
\text { RW-730 }\end{array}$ & $\begin{array}{l}\text { Scrophulariace } \\
\text { ae }\end{array}$ & Tonch & $\mathrm{H}$ & St, L & $\begin{array}{l}\text { Blood purifier or } \\
\text { oxygenation, forage. }\end{array}$ & 0.5 & 6 & 0.04 & $\mathrm{Di}$ & Or & $\begin{array}{l}1 \bullet 2 \backsim 3 \backsim 4 \bullet 5 \bullet 6 \bullet \\
7 \bullet 8 \bullet 9 \bullet 10 \bullet 11 \bullet \\
12 \bullet 13 \bullet 14 \bullet\end{array}$ \\
\hline $\begin{array}{l}\text { Datura } \\
\text { stramonium L. } \\
\text { RW-667 }\end{array}$ & Solanaceae & Tandouro & $\mathrm{H}$ & $\mathrm{S}, \mathrm{FI}, \mathrm{L}$ & $\begin{array}{l}\text { Anti dandruf, tooth } \\
\text { ache, ear ache, } \\
\text { boils, remove worms } \\
\text { from wounded sites. }\end{array}$ & 2.64 & 43 & 0.29 & $\begin{array}{l}\mathrm{Po}, \mathrm{J} \\
\mathrm{Pa}\end{array}$ & Or/E & $\begin{array}{l}1 \square 2 \bullet 3 \sqcap 4 \square 5 \square 6 \bullet \\
7 \bullet 8 \square 9 \bullet 10 \bullet 11 \bullet \\
12 \bullet 13 \bullet 14 \square\end{array}$ \\
\hline $\begin{array}{l}\text { Daphne } \\
\text { mucronata Royle } \\
\text { RW-666 }\end{array}$ & $\begin{array}{l}\text { Thymelaeacea } \\
\mathrm{e}\end{array}$ & Nick & Sh & $\mathrm{F}, \mathrm{L}$ & $\begin{array}{l}\text { Eye problem, } \\
\text { toothache, break big } \\
\text { stones. }\end{array}$ & 0.8 & 41 & 0.28 & Di, Ex & Or & $\begin{array}{l}1 \bullet 2 \bullet 3 \bullet 4 \square 5 \bullet 6 \square \\
7 \bullet 8 \bullet 9 \bullet 10 \square 11 \bullet \\
12 \bullet 13 \bullet 14 \bullet\end{array}$ \\
\hline $\begin{array}{l}\text { Foeniculum } \\
\text { vulgare Mill. } \\
\text { RW-675 }\end{array}$ & Umbelliferae & Nerlay gizari & $\mathrm{H}$ & $\mathrm{F}$ & $\begin{array}{l}\text { Eyesight, Aromatic, } \\
\text { Carminative, } \\
\text { stomachic, digestive, } \\
\text { forage. }\end{array}$ & 0.62 & 30 & 0.20 & $\mathrm{De}$ & Or & $\begin{array}{l}1 \bullet 2 \bullet 3 \bullet 4 \bullet 5 \bullet 6 \square \\
7 \square 8 \bullet 9 \square 10 \bullet 11 \bullet \\
12 \bullet 13 \bullet 14 \bullet\end{array}$ \\
\hline $\begin{array}{l}\text { Urtica dioica L. } \\
\text { RW-729 }\end{array}$ & Urticaceae & Jomi & $\mathrm{H}$ & $L$ & $\begin{array}{l}\text { Arthritis, swollen } \\
\text { parts, nausea, } \\
\text { vegetable. }\end{array}$ & 1.5 & 35 & 0.23 & $\mathrm{~Pa}, \mathrm{~J}$ & Or & $\begin{array}{l}1 \bullet 2 \square 3 \square 4 \square 5 \bullet 6 \bullet \\
7 \bullet 8 \bullet 9 \bullet 10 \bullet 11 \bullet \\
12 \bullet 13 \bullet 14 \square\end{array}$ \\
\hline $\begin{array}{l}\text { Viola pilosa } \\
\text { Blume } \\
\text { RW-731 }\end{array}$ & Violaceae & Lilio & $\mathrm{H}$ & $\mathrm{F}, \mathrm{L}$ & $\begin{array}{l}\text { Cough, anti- } \\
\text { diarrheal, fever, used } \\
\text { for blood reduction, } \\
\text { pneumonia, edible, } \\
\text { asthma, }\end{array}$ & 0.5 & 12 & 0.08 & Po & Or & $\begin{array}{l}1 \bullet 2 \bullet 3 \bullet 4 \bullet 5 \bullet 6 \bullet \\
7 \bullet 8 \bullet 9 \bullet 10 \bullet 11 \bullet \\
12 \bullet 13 \bullet 14 \bullet\end{array}$ \\
\hline
\end{tabular}




\begin{tabular}{|l|l|l|l|l|l|l|l|l|l|}
\hline & & & & $\begin{array}{l}\text { constipation, } \\
\text { stomachic, forage. }\end{array}$ & & & \\
\hline $\begin{array}{l}\text { Vitis vinifera L. } \\
\text { RW-732 }\end{array}$ & Vitaceae & Kasheelzach & WC & F & $\begin{array}{l}\text { Kidney stone, } \\
\text { produce blood, } \\
\text { constipation, cough, } \\
\text { smoking, improve } \\
\text { urine muscle, } \\
\text { remove intestinal } \\
\text { worms, hair } \\
\text { coloration leaves use } \\
\text { for forage, fruit has } \\
\text { high economic value. }\end{array}$ & 1.30 & 49 & 0.33 \\
\hline $\begin{array}{l}\text { Tribulus terrestris } \\
\text { L. Zygophyllacea } \\
\text { RW-728 }\end{array}$ & e & Kunay & H & F & $\begin{array}{l}\text { Pimples, menses, } \\
\text { blood pressure, } \\
\text { kidney stone. }\end{array}$ & 0.88 & 31 & 0.21 \\
\hline
\end{tabular}

$\mathrm{L}=$ leaf, $\mathrm{R}=$ root, $\mathrm{B}=$ bark, $\mathrm{S}=$ shoot, $\mathrm{St}=\mathrm{Stem}, \mathrm{F}=\mathrm{Fruit}, \mathrm{Fl}=\mathrm{Flower}, \mathrm{La}=$ latex, $\mathrm{G}=$ gum, $\mathrm{Wp}=$ whole plant, $\mathrm{C}=$ cone, $\mathrm{Pa}=\mathrm{paste}, \mathrm{P}=$ poultice, $\mathrm{Po}=\mathrm{Powder}, \mathrm{De}=\mathrm{Decoction}, \mathrm{In}=$ infusion. As=ash, Fu=fume, Di=Direct, Ex=extract, Sa=saag, J=juice, Sh=shrub, H=herb, T=tree, WC=woody climber $E=$ external, Or=oral, To=topical, In=inhalation, SC= seed coat, RW=Rawalpindi, $\square=$ Reported with similar Use, $\mathbf{\square = R e p o r t e d ~ w i t h ~ d i s s i m i l a r ~ u s e , ~} \bullet=$ Not Reported, FC=Frequency of Citation, RFC=Relative Frequency of Citation, UV=Use Value,UVI=Use Value Index.

1=(Qureshi et al., 2007); 2=(Shinwari and Gilani, 2003); 3=(Siddiqui, 2015); 4=(Abbas et al., 2014.); $5=($ Khan et al., 2013); 6=(Afzal et al., 2009); 7=(Awan et al., 2013); 8=(Shedayi et al., 2014); $\mathbf{9 = ( ~ H u s s a i n ~ e t ~ a l . , ~ 2 0 1 1 ) ; ~ 1 0 = ( K h a n ~ a n d ~ K h a t o o n , ~ 2 0 0 7 ) ; ~ 1 1 = ( K h a n ~ e t ~ a l . , ~ 2 0 1 1 ) ; ~ 1 2 = ( F a h a d ~ a n d ~ B a n o , ~ 2 0 1 2 ) ; ~ 1 3 = ( B a n o ~ e t ~ a l . , ~ 2 0 1 4 ) ; ~ 1 4 = ( S h e d a y i ~ a n d ~ G u l s h a n , ~}$ 2012). 

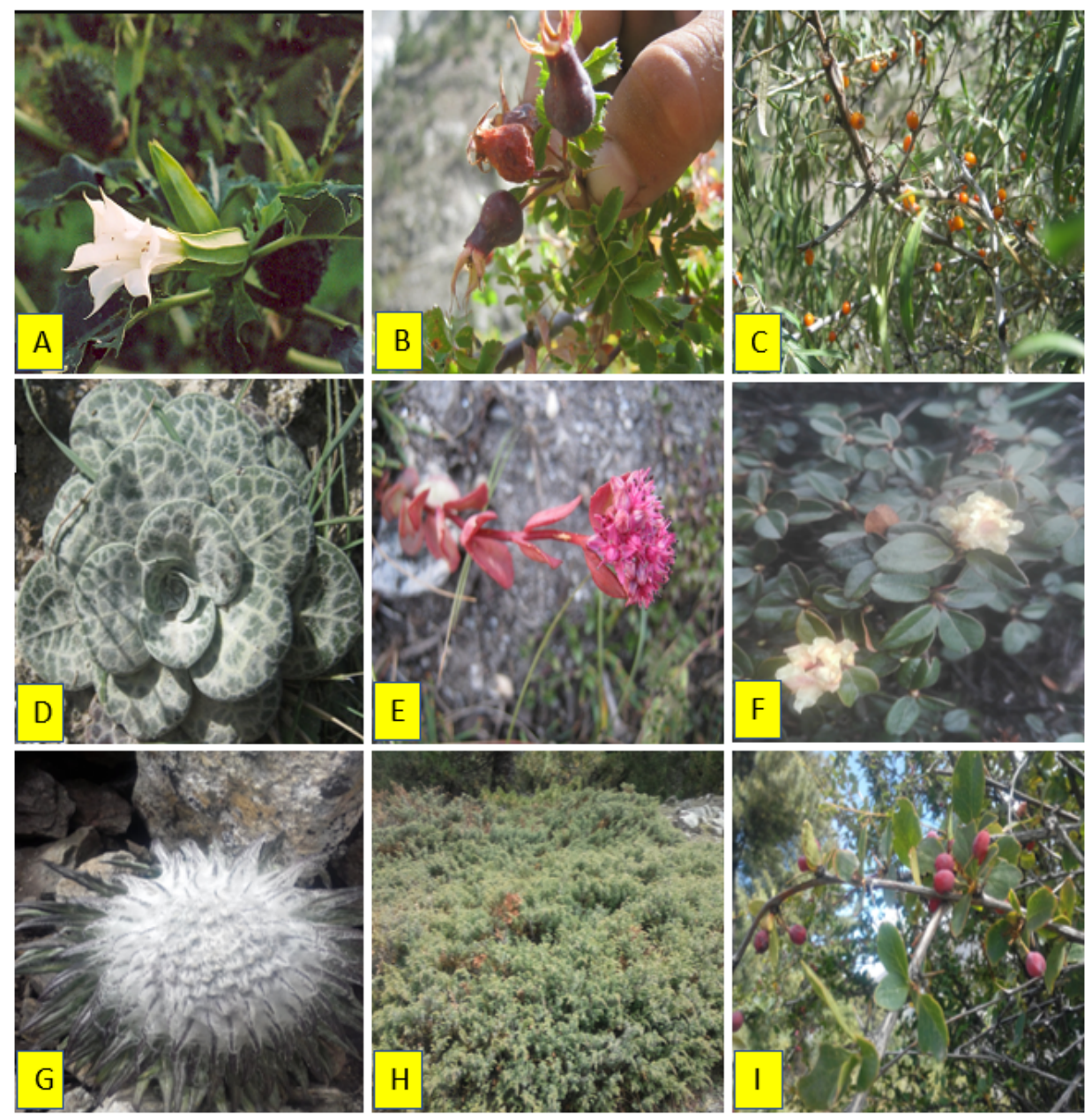

Fig. 2. Medicinal plants collected from the study area. (a) Berberis lyceum, (b) Rosa webbiana, (c) Datura stramonium (d) Juniperus communis (e) Limonium cabulicum (f) Saussurea gossypiphora, (g) Rhododendron anthopogon (h) Hylotelephium ewersii (i) Hippophae rhamnoides
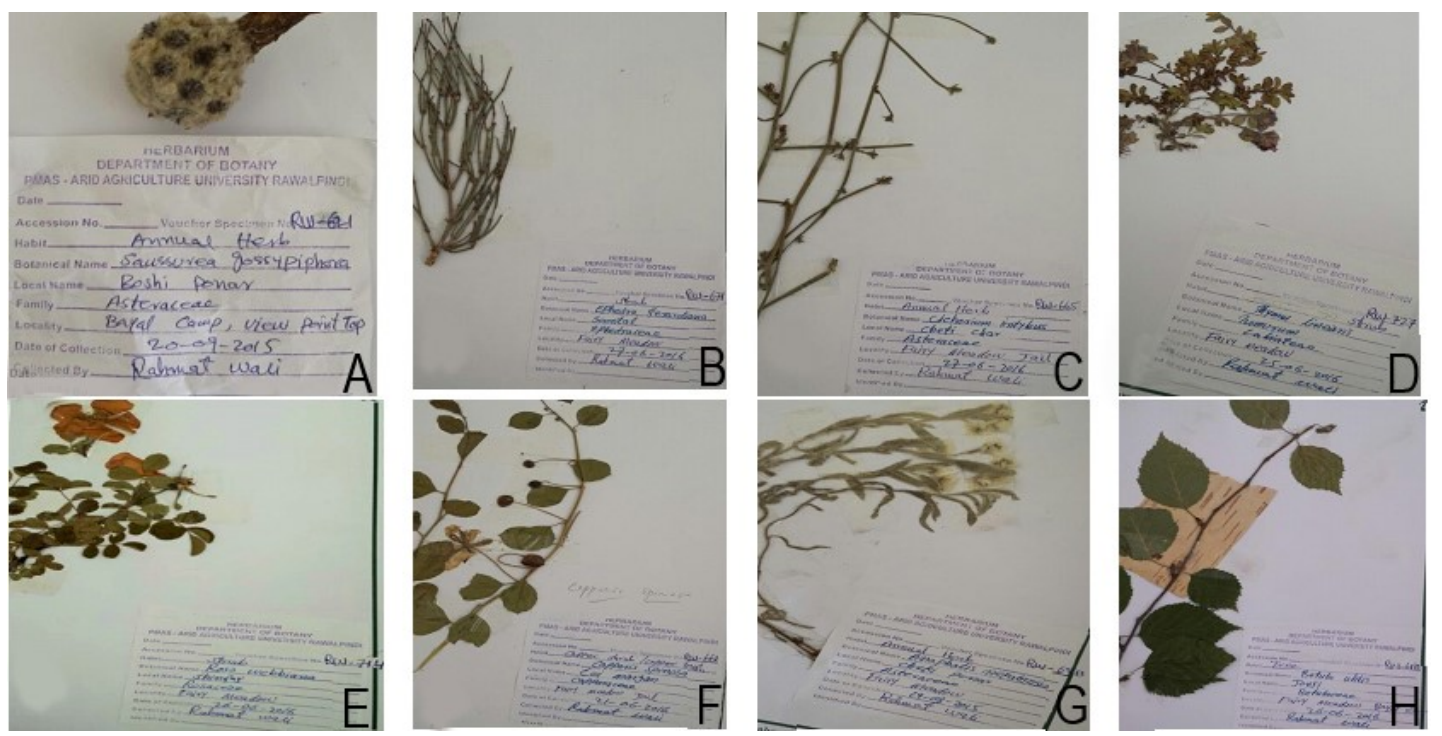

Fig. 3. Medicinal plants with voucher number. (A) Saussurea gossypiphora, (B) Ephedra gerardiana, (C) Cichorium intybus, (D) Thymus linearis, (E) Rosa webbiana, (F) Capparis spinosa, (G) Anaphalis nepalensis and (H) Betula utilis 


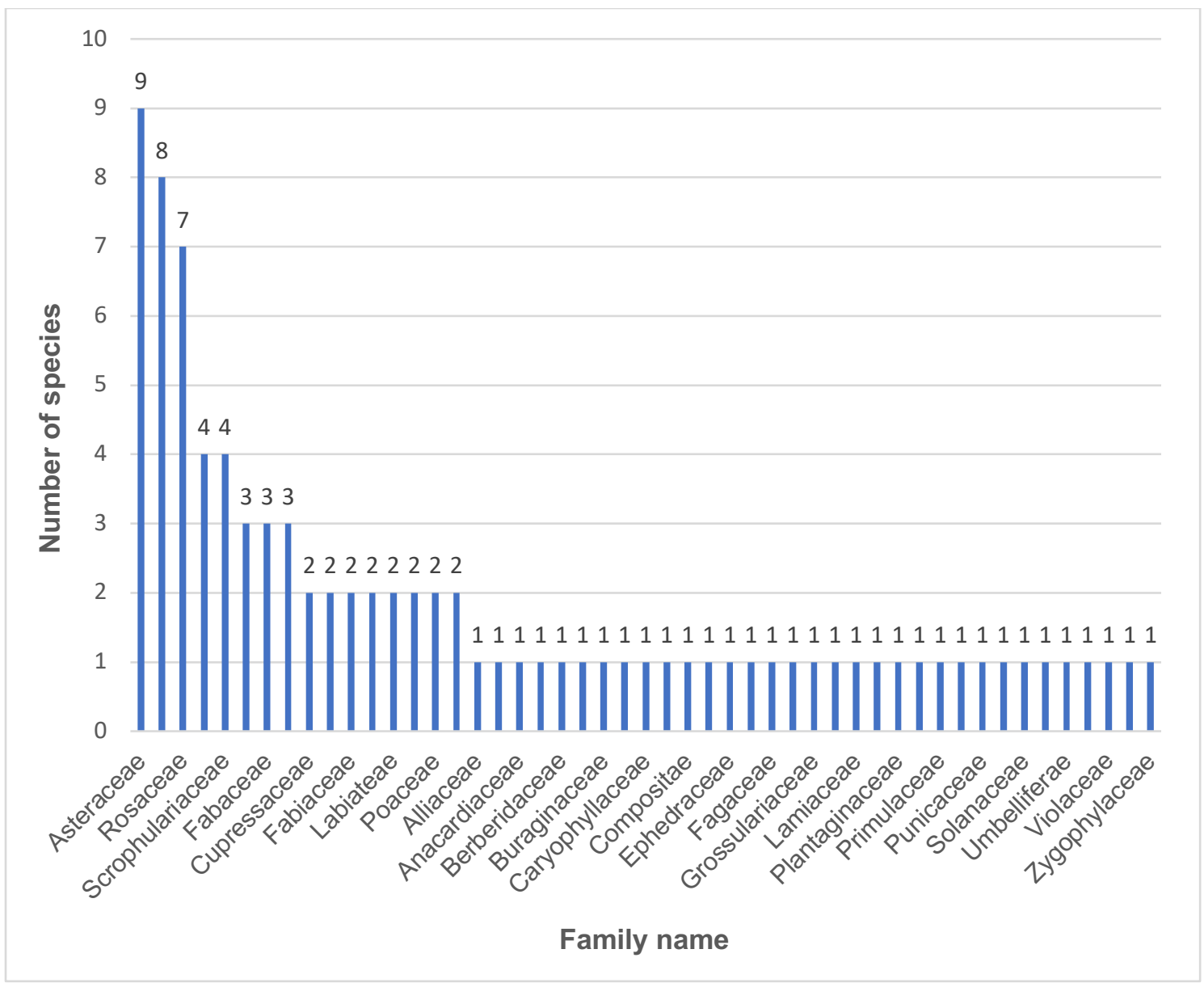

Fig. 4. Dominant plant families used in the study area.

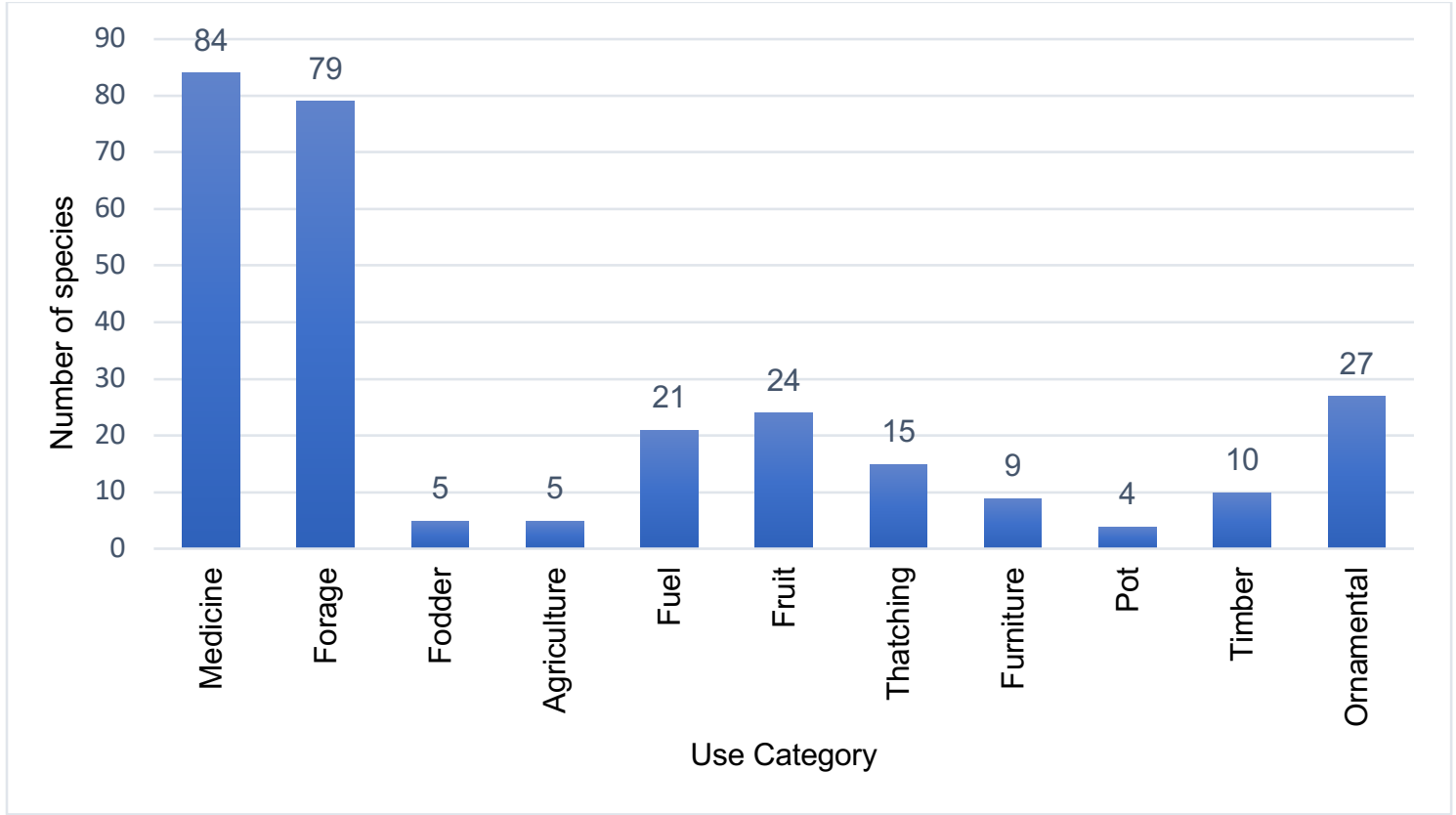

Fig. 5. Use categories of plants. 


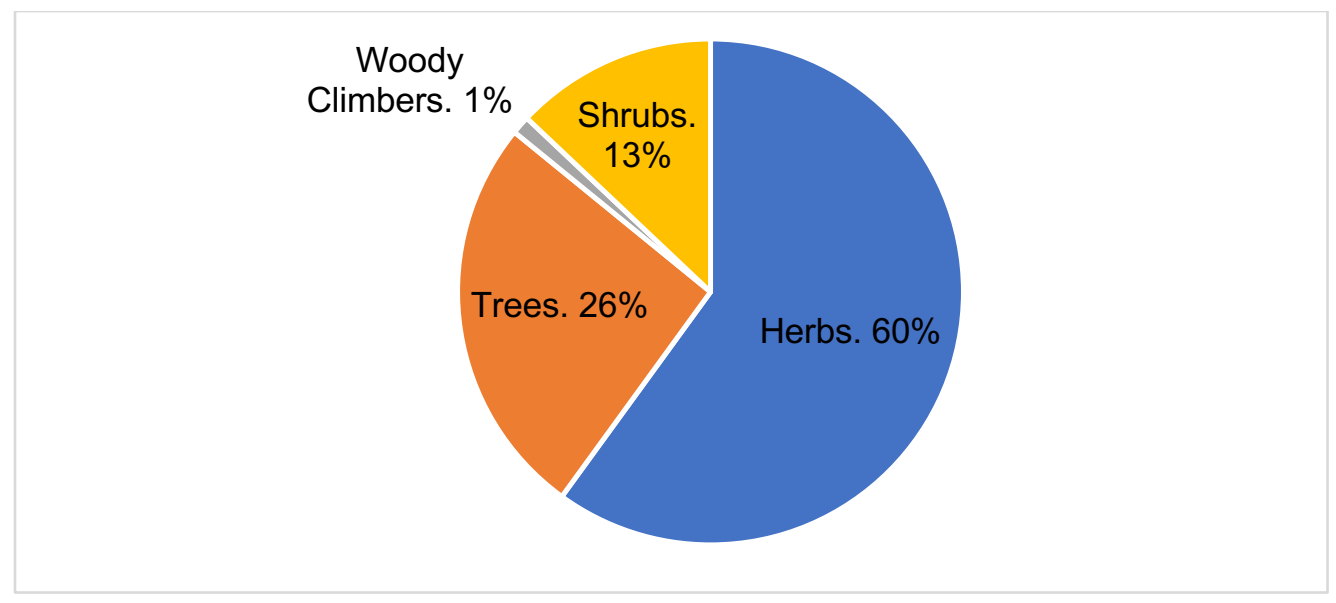

Fig. 6. Dominant life forms of medicinal plants.

\section{Cultivated plants. $14 \%$}

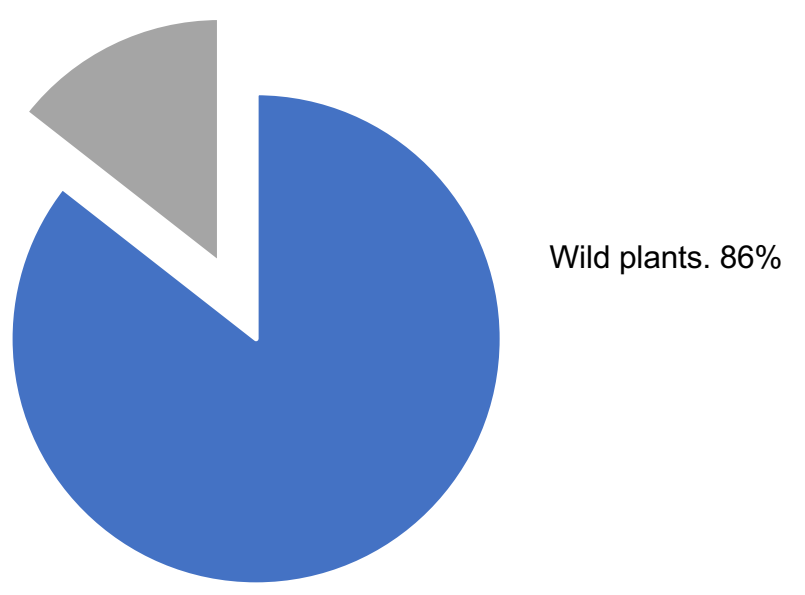

Fig. 7. Percentage of wild and cultivated medicinal plants.

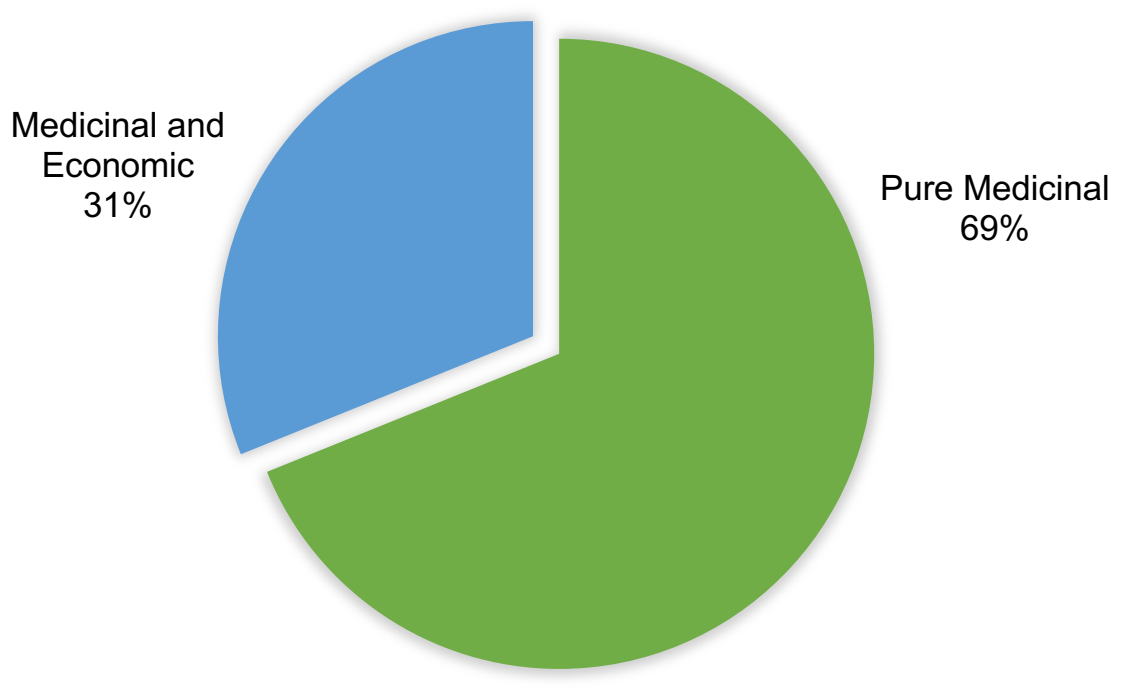

Fig. 8. Percentage of pure medicinal and both medicinal and economic important plants. 


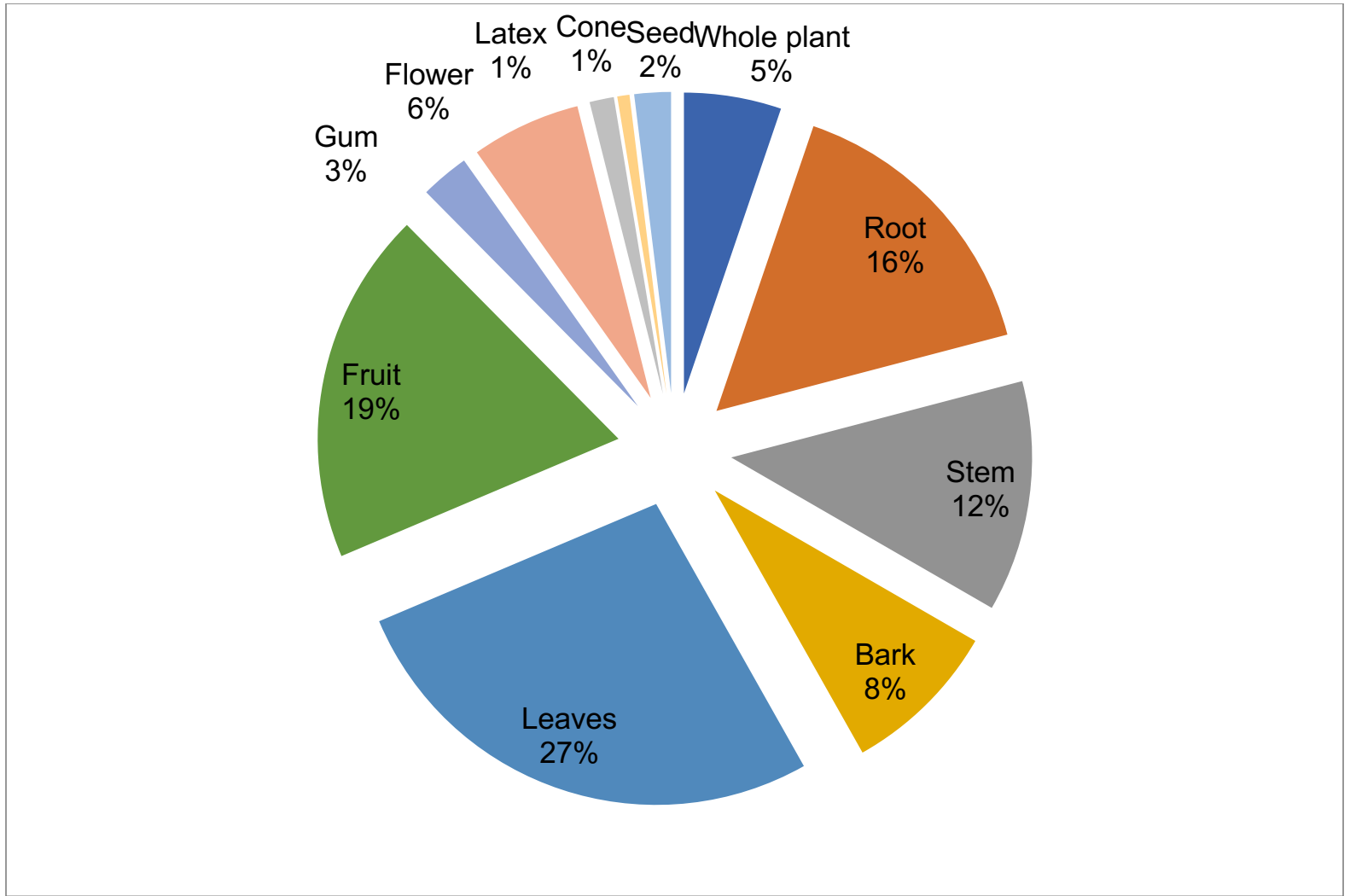

Fig. 9. Plant parts used in traditional medicine.

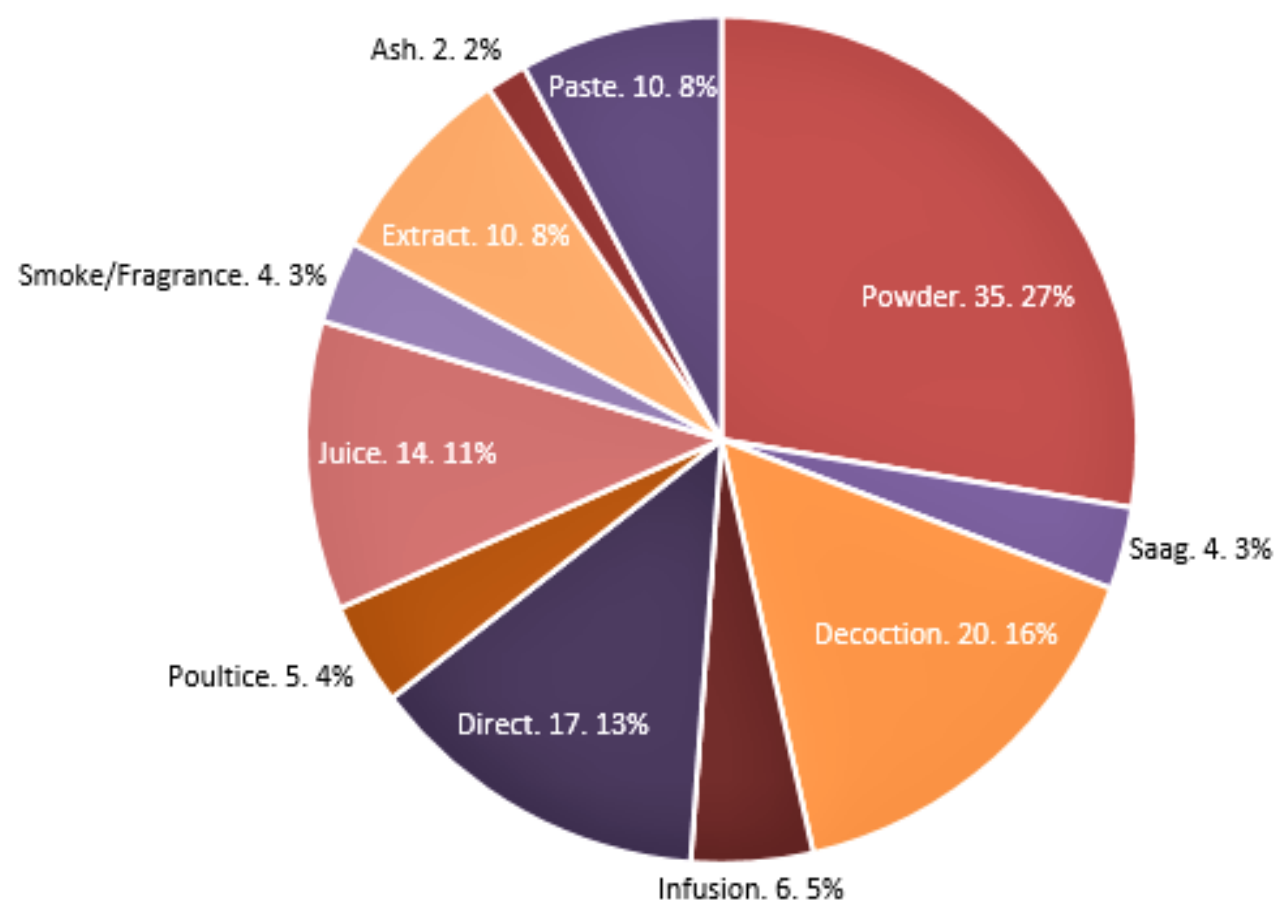

Fig. 10. Percent distribution of different herbal preparations. 


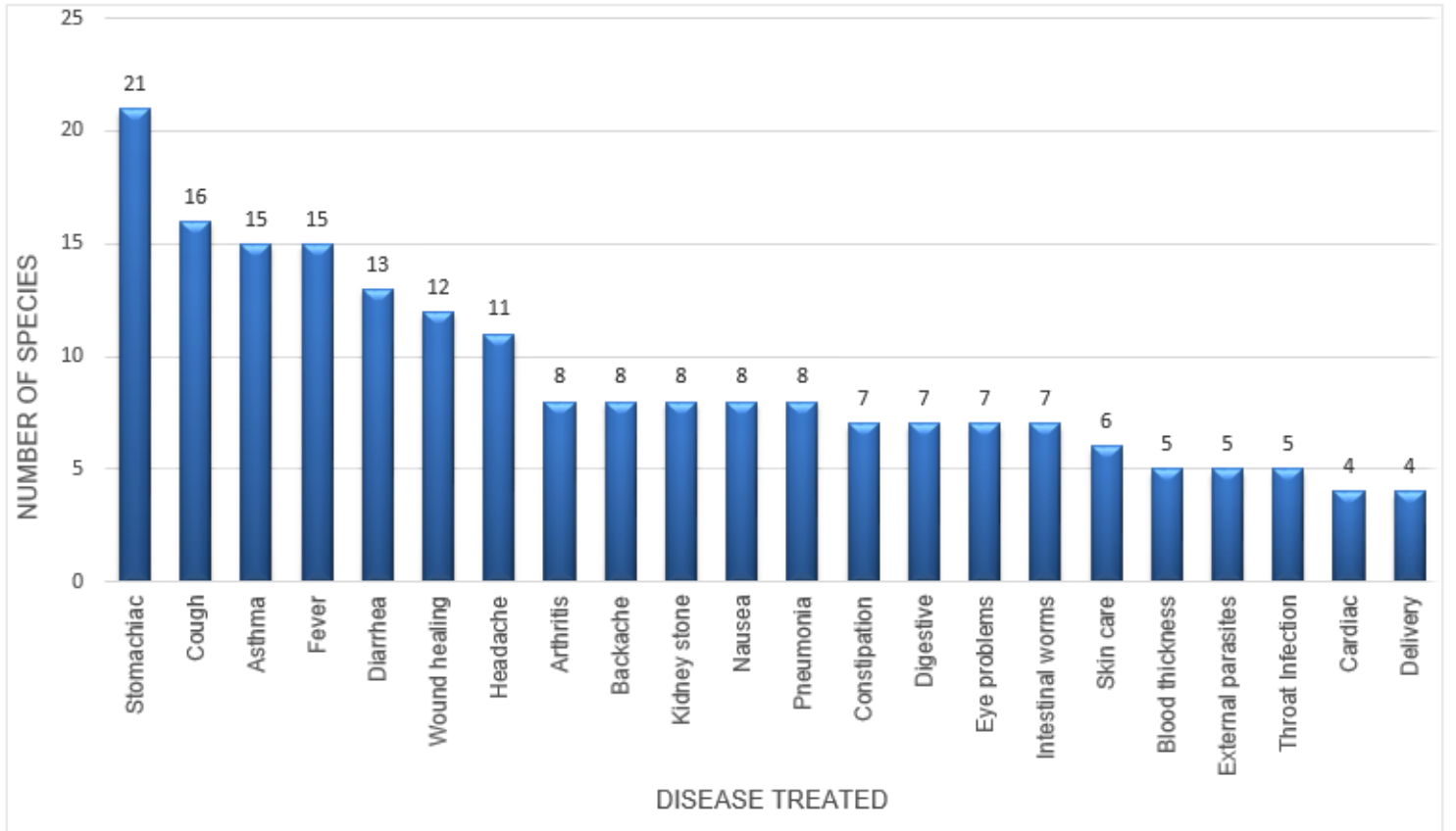

Fig. 11. Most common ailments treated with medicinal plant species.

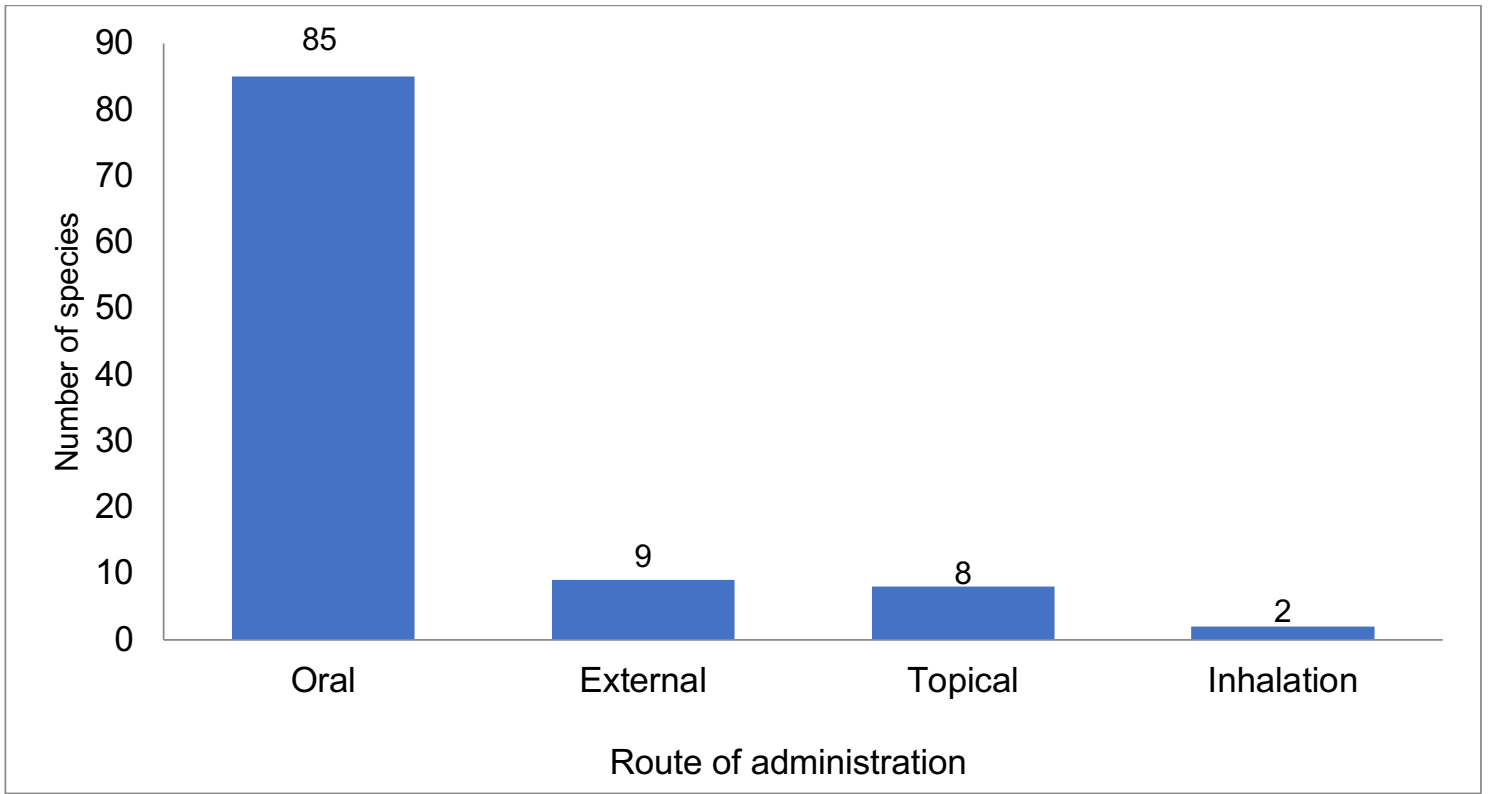

Fig. 12. Mode of administration of herbal preparations.

Thirty-one out of 90 plant species were reported for the first time in this study, either with new uses (28 plants) or new report (3 plants). The newly reported medicinal plant species to the world of ethnobotany include Allium gilgitensis, Astragalus gilgitensis and Pedicularia flava. The new ethnomedicinal data was compared with the previous studies from the neighboring areas of district Diamir and other countries (Khan and Khatoon, 2007; Telkes, 2011) showing very few similar uses while most of the documented plants have unique uses. Adiantum raddianum was reported to cure scabies by John and Mahesh (2007), while the same species in our study was used for fever, diarrhea, and as forage. Anacyclus pyrethrum species was reported from Jorden to cure colitis (colon inflammation) by Afifi and Ab-Irmaileh (2000), while in this study it was used for sore throat, eye wash, toothache and forage. Similarly, Asparagus officinalis was reported as used for common cold, cough, and influenza by Afifi and Ab-Irmaileh, (2000), while uses in our study included to cure kidney problem, used to cure the diabetic persons, digestive, and in maintenance of blood glucose level. The comparative details (reported and novel uses) of all 31 plants species are given in Table 3. 
Table 3. Medicinal Plants with novel and already reported uses

\begin{tabular}{|c|c|c|c|}
\hline Scientific Name & Novel reports & Already reported uses & References \\
\hline $\begin{array}{l}\text { Adiantum } \\
\text { raddianum }\end{array}$ & $\begin{array}{l}\text { Fever, diarrhea, } \\
\text { forage. }\end{array}$ & $\begin{array}{l}\text { Plant extract is applied on the surface } \\
\text { of body to cure scabies. }\end{array}$ & $\begin{array}{l}\text { John and Mahesh, } \\
\text { 2007. India. }\end{array}$ \\
\hline Allium gilgitensis & $\begin{array}{l}\text { Pain, headache, } \\
\text { vegetable, forage. }\end{array}$ & & \\
\hline $\begin{array}{l}\text { Anacyclus } \\
\text { pyrethrum }\end{array}$ & $\begin{array}{l}\text { Sore throat, eye wash, } \\
\text { toothache and forage. }\end{array}$ & $\begin{array}{l}\text { Stem is used to cure colitis (colon } \\
\text { inflammation). }\end{array}$ & $\begin{array}{l}\text { Afifi and Ab-Irmaileh, } \\
\text { 2000. Jordon }\end{array}$ \\
\hline $\begin{array}{l}\text { Artemisia } \\
\text { glacialis }\end{array}$ & $\begin{array}{l}\text { Diarrhea, nausea, } \\
\text { intestinal worms, } \\
\text { helpful in pregnancy. }\end{array}$ & Aerial parts used as digestive & $\begin{array}{l}\text { Pieroni and Giusti. } \\
\text { 2009. Varaita valley, } \\
\text { Piedmont, Itlay }\end{array}$ \\
\hline $\begin{array}{l}\text { Asparagus } \\
\text { officinalis }\end{array}$ & $\begin{array}{l}\text { Anti-inflammatory, } \\
\text { diabetes, antioxidant, } \\
\text { digestive, anti-cancer, } \\
\text { maintains blood } \\
\text { glucose level, forage. }\end{array}$ & common cold, cough, influenza & $\begin{array}{l}\text { Afifi and } \\
\text { Abulrmaileh, } 2000 . \\
\text { Jordon. }\end{array}$ \\
\hline Aster himalaicus & $\begin{array}{l}\text { Stomachic, aromatic, } \\
\text { forage. }\end{array}$ & Root decoction is used for dysentery & $\begin{array}{l}\text { Bano et al., } 2014 . \\
\text { Gilgit Baltistan, } \\
\text { Pakistan }\end{array}$ \\
\hline $\begin{array}{l}\text { Astragalus } \\
\text { gilgitensis }\end{array}$ & Digestive, Forage. & ---- & ---- \\
\hline Bistorta vivipara & $\begin{array}{l}\text { Fruit edible, throat } \\
\text { infection, root for piles, } \\
\text { forage. }\end{array}$ & $\begin{array}{l}\text { Fried seeds are used in checking blood } \\
\text { dysentery }\end{array}$ & $\begin{array}{l}\text { Jalal and Kumar, } \\
\text { 2006. Nepal }\end{array}$ \\
\hline $\begin{array}{l}\text { Cicer } \\
\text { microphyllum }\end{array}$ & Fruit edible, forage. & $\begin{array}{l}\text { Whole plant is used for increasing milk } \\
\text { production and as general tonic for } \\
\text { cows. }\end{array}$ & $\begin{array}{l}\text { Khan and Khatoon, } \\
\text { 2008. Gilgit Baltistan }\end{array}$ \\
\hline $\begin{array}{l}\text { Equisetum } \\
\text { hyemale }\end{array}$ & $\begin{array}{l}\text { Kidney problems, skin } \\
\text { and mouth dryness, } \\
\text { forage. }\end{array}$ & $\begin{array}{l}\text { Nutrients in the plant may cause skin, } \\
\text { hair, teeth and nails to become } \\
\text { stronger. Used as ingredient of } \\
\text { shampoo, soap and skin care } \\
\text { products. Also, used to boost milk } \\
\text { production in cows. }\end{array}$ & $\begin{array}{l}\text { Telkes, } 2011 . \\
\text { America }\end{array}$ \\
\hline \multirow[t]{3}{*}{ Ficus palmata } & \multirow[t]{3}{*}{$\begin{array}{l}\text { Asthma, Obstruction in } \\
\text { liver and spleen, latex } \\
\text { used for blisters and } \\
\text { healing }\end{array}$} & $\begin{array}{l}\text { Fruit edible, leaves as fodder and } \\
\text { wood used for fuel, fruit paste used in } \\
\text { ringworm and skin diseases. }\end{array}$ & $\begin{array}{l}\text { Thapa, } 2001 . \\
\text { Manandhar, } 2002 . \\
\text { Pant and Panta, } \\
\text { 2004. Nepal. }\end{array}$ \\
\hline & & $\begin{array}{l}\text { The fruit is demulcent, emollient, } \\
\text { laxative and poultice. It is used as a } \\
\text { part of the diet in the treatment of } \\
\text { constipation and diseases of the lungs } \\
\text { and bladder. The latex of the plant is } \\
\text { used to take out spines lodged deeply } \\
\text { in the flesh. }\end{array}$ & Haq, 2012. Pakistan \\
\hline & & $\begin{array}{l}\text { Latex is applied to heal wounds and } \\
\text { cuts }\end{array}$ & $\begin{array}{l}\text { Nautiyal et al., } 2008 . \\
\text { India }\end{array}$ \\
\hline \multirow[t]{2}{*}{ Gentiana kurroo } & \multirow{2}{*}{$\begin{array}{l}\text { Healing of wounds, } \\
\text { removal of dead cells, } \\
\text { boils, headache, cold, } \\
\text { fever, malaria, forage. }\end{array}$} & $\begin{array}{l}\text { Roots are dried to make powder and } \\
\text { taken along desi ghee for } \\
\text { Stomachache. }\end{array}$ & $\begin{array}{l}\text { Adnan et al., } 2015 . \\
\text { Pakistan }\end{array}$ \\
\hline & & $\begin{array}{l}\text { Skin disease, leprosy, leucoderma, } \\
\text { constipation }\end{array}$ & $\begin{array}{l}\text { Bano et al., } 2014 . \\
\text { Gilgit Baltistan, } \\
\text { Pakistan }\end{array}$ \\
\hline $\begin{array}{l}\text { Geranium } \\
\text { pratense }\end{array}$ & $\begin{array}{l}\text { After delivery to reduce } \\
\text { abdominal pain, } \\
\text { backache, forage and } \\
\text { milk production in } \\
\text { cattle. }\end{array}$ & $\begin{array}{l}\text { These plants are used for internal and } \\
\text { external wounds, swellings, } \\
\text { inflammations, tumors, bleeding, } \\
\text { uterus problems and nerve problems. }\end{array}$ & $\begin{array}{l}\text { Khan and Khatoon, } \\
\text { 2008. Gilgit Baltistan }\end{array}$ \\
\hline $\begin{array}{l}\text { Gnaphalium } \\
\text { spp. }\end{array}$ & $\begin{array}{l}\text { Constipation, menses, } \\
\text { sciatica, forage. }\end{array}$ & $\begin{array}{l}\text { Aerial parts used for hypertension } \\
\text { and ulcers. }\end{array}$ & $\begin{array}{l}\text { Zheng et al., } 2013 . \\
\text { China }\end{array}$ \\
\hline $\begin{array}{l}\text { Hylotelephium } \\
\text { ewersi }\end{array}$ & $\begin{array}{l}\text { Constipation, } \\
\text { Stomachic. }\end{array}$ & Aerial parts used as appetizer & $\begin{array}{l}\text { Bano et al., } 2014 . \\
\text { Gilgit Baltistan, } \\
\text { Pakistan }\end{array}$ \\
\hline
\end{tabular}




\begin{tabular}{|c|c|c|c|}
\hline $\begin{array}{l}\text { Limonium } \\
\text { cabulicum }\end{array}$ & $\begin{array}{l}\text { Asthma, pneumonia, } \\
\text { tuberculosis, bleeding } \\
\text { gums, piles, thought to } \\
\text { be used for thousand } \\
\text { diseases, forage. }\end{array}$ & $\begin{array}{l}\text { Fresh leaves boiled in water containing } \\
\text { sugar and used as tea. }\end{array}$ & $\begin{array}{l}\text { Ahmad and Pieroni, } \\
\text { 2016. Thakht-e- } \\
\text { Sulaiman Hills, } \\
\text { North-West Pakistan }\end{array}$ \\
\hline \multirow[t]{2}{*}{$\begin{array}{l}\text { Malus } \\
\text { domestica }\end{array}$} & \multirow[t]{2}{*}{$\begin{array}{l}\text { Blood purification, } \\
\text { Edible, health tonic, } \\
\text { financially important as } \\
\text { fruit sold in market. }\end{array}$} & $\begin{array}{l}\text { Decoction with Ficus carica } \\
\text { pseudofruits, aerial parts of Malva } \\
\text { sylvestris and Verbascus thapsus } \\
\text { leaves used as antitussive. }\end{array}$ & $\begin{array}{l}\text { Pieroni et al., } 2002 . \\
\text { Italy. }\end{array}$ \\
\hline & & Tea for common cold and cough. & $\begin{array}{l}\text { Saric-Kundalic et al., } \\
\text { 2010. Bosnia }\end{array}$ \\
\hline $\begin{array}{l}\text { Pedicularis } \\
\text { bicornata }\end{array}$ & $\begin{array}{l}\text { General body pain, } \\
\text { stomachic, sedative, } \\
\text { forage. }\end{array}$ & $\begin{array}{l}\text { Leaf juice used to stop bleeding of } \\
\text { cuts. }\end{array}$ & $\begin{array}{l}\text { Nautiyal et al., } 2008 . \\
\text { India }\end{array}$ \\
\hline Pedicularis flava & Asthma, forage. & ---- & ---- \\
\hline $\begin{array}{l}\text { Pedicularis } \\
\text { pectinate }\end{array}$ & $\begin{array}{l}\text { Stomachic, cardiac, } \\
\text { forage. }\end{array}$ & $\begin{array}{l}\text { Pounced leaves are given for } \\
\text { haemoptysis. }\end{array}$ & $\begin{array}{l}\text { Wagay, 2016. North } \\
\text { Kashmir }\end{array}$ \\
\hline Picea smithiana & $\begin{array}{l}\text { Gum is used for } \\
\text { Asthma, diarrhea, } \\
\text { tuberculosis, diabetes, } \\
\text { join broken pots, hair } \\
\text { removing. }\end{array}$ & $\begin{array}{l}\text { Resin is used for joining different } \\
\text { things as well as used for heart } \\
\text { problems. Wood is a chief source of } \\
\text { timber and fuel }\end{array}$ & $\begin{array}{l}\text { Khan and Khatoon, } \\
\text { 2007. Gilgit Baltistan }\end{array}$ \\
\hline \multirow[t]{2}{*}{ Prunus persica } & \multirow[t]{2}{*}{$\begin{array}{l}\text { Seed coat used for eye } \\
\text { problem, fruit edible }\end{array}$} & $\begin{array}{l}\text { The dried fruits and ginger are crushed } \\
\text { into powder. This powder is mixed with } \\
\text { honey and eaten for body cooling and } \\
\text { diabetes. The resin is boiled in water, } \\
\text { cool down and used for earache and } \\
\text { deafness. }\end{array}$ & Haq, 2012. Pakistan \\
\hline & & $\begin{array}{l}\text { Seed oil used in massage for warmth } \\
\text { during extreme cold season. }\end{array}$ & $\begin{array}{l}\text { Nautiyal et al., } 2008 . \\
\text { ndia }\end{array}$ \\
\hline Quercus incana & $\begin{array}{l}\text { Furniture, cattle forage, } \\
\text { fuel wood, thatching, } \\
\text { wood/timber sold in } \\
\text { market. }\end{array}$ & $\begin{array}{l}\text { Fruits is used as astringent, diuretic, } \\
\text { diarrhea and asthma. }\end{array}$ & Haq, 2012. Pakistan \\
\hline $\begin{array}{l}\text { Rheum } \\
\text { webbianum }\end{array}$ & $\begin{array}{l}\text { Hair coloring, blood } \\
\text { purifier, stomachic, } \\
\text { dyspepsia, tonic for } \\
\text { goat, sheep, forage. }\end{array}$ & $\begin{array}{l}\text { Roots crushed, and paste applied on } \\
\text { wounds and cuts }\end{array}$ & $\begin{array}{l}\text { Nautiyal et al., } 2008 . \\
\text { India }\end{array}$ \\
\hline Rubus ulmifolius & Hepatitis, Edible. & $\begin{array}{l}\text { Fruits are edible and carminative. } \\
\text { Unripe fruit are used as tonic and } \\
\text { aphrodisiac. Roots and leaves are } \\
\text { used for the treatment f skin diseases. }\end{array}$ & Haq, 2012. Pakistan \\
\hline $\begin{array}{l}\text { Saussurea } \\
\text { gossypiphora }\end{array}$ & $\begin{array}{l}\text { Asthma, pneumonia, } \\
\text { stomach problem, flue, } \\
\text { headache, improve } \\
\text { circulation, menstrual } \\
\text { cycle, decoction used } \\
\text { to expel umbilical cord } \\
\text { easily. }\end{array}$ & Flower is used for fever & Kala, 2005. India \\
\hline \multirow[t]{2}{*}{ Silene vulgaris } & \multirow{2}{*}{$\begin{array}{l}\text { Cattle forage, intestinal } \\
\text { worms, kidney } \\
\text { problem. }\end{array}$} & Aerial parts used for female disorders. & $\begin{array}{l}\text { Saric-Kundalic et al., } \\
\text { 2010. Bosnia }\end{array}$ \\
\hline & & $\begin{array}{l}\text { Leaves juice used as Ophthalmia, } \\
\text { stomachic, emollient. }\end{array}$ & $\begin{array}{l}\text { Bano et al., } 2014 . \\
\text { Gilgit Baltistan, } \\
\text { Pakistan }\end{array}$ \\
\hline \multirow[t]{2}{*}{ Viola pilosa } & \multirow{2}{*}{$\begin{array}{l}\text { Cough, anti-diarrheal, } \\
\text { fever, maintains the } \\
\text { blood in body in normal } \\
\text { range, pneumonia, } \\
\text { edible, asthma, } \\
\text { constipation, } \\
\text { stomachic, forage. }\end{array}$} & Used as expectorant. & $\begin{array}{l}\text { Saric-Kundalic et al., } \\
\text { 2010. Bosnia. }\end{array}$ \\
\hline & & $\begin{array}{l}\text { Fresh flowers are boiled in water and } \\
\text { decoction is prepared. The decoction is } \\
\text { used as tea to cure fever, cough and } \\
\text { cold. }\end{array}$ & Uniyal et al., 2006. \\
\hline Vitis vinifera & $\begin{array}{l}\text { Kidney stone, produce } \\
\text { blood, constipation, }\end{array}$ & $\begin{array}{l}\text { General weakness. Fruit is used for } \\
\text { abdominal inflammation. }\end{array}$ & $\begin{array}{l}\text { Afifi and Abu-Irmaileh, } \\
\text { 2000. Jordon. }\end{array}$ \\
\hline
\end{tabular}




\begin{tabular}{|c|c|c|c|}
\hline & $\begin{array}{l}\text { cough, smoking, } \\
\text { improve urine muscle, } \\
\text { remove intestinal } \\
\text { worms, hair coloration } \\
\text { leaves use for forage, } \\
\text { fruit is sold in market } \\
\text { for financial aid. }\end{array}$ & $\begin{array}{l}\text { Tea for blood purification, renal and } \\
\text { bladder ailments. }\end{array}$ & $\begin{array}{l}\text { Saric-Kundalic et al., } \\
\text { 2010. Bosnia }\end{array}$ \\
\hline $\begin{array}{l}\text { Xanthium } \\
\text { stramonium }\end{array}$ & Swelling, boils. & $\begin{array}{l}\text { Fruit is demulcent and cooling, used in } \\
\text { smallpox. Leaves decoction is } \\
\text { recommended in long standing } \\
\text { malarial fever. An infusion of the plant } \\
\text { has been used in the treatment of } \\
\text { rheumatism, diseased kidneys and } \\
\text { tuberculosis. A decoction of the root } \\
\text { has been used in the treatment of high } \\
\text { fevers and to help a woman expel the } \\
\text { afterbirth. A decoction of the seeds has } \\
\text { been used in the treatment of bladder } \\
\text { complaints. A poultice of the powdered } \\
\text { seed has been applied as a salve on } \\
\text { open sores. }\end{array}$ & Haq, 2012. Pakistan \\
\hline \multirow[t]{2}{*}{ Zea mays } & \multirow{2}{*}{$\begin{array}{l}\text { Paste applied on } \\
\text { swelling parts, blisters, } \\
\text { as food, fodder, } \\
\text { economic }\end{array}$} & $\begin{array}{l}\text { Tea for blood purification, renal and } \\
\text { bladder ailments }\end{array}$ & $\begin{array}{l}\text { Saric-Kundalic et al., } \\
\text { 2010. Bosnia }\end{array}$ \\
\hline & & $\begin{array}{l}\text { Maize is the second chief source of } \\
\text { food and straw and its grain is also } \\
\text { used medicinally for dysentery, } \\
\text { jaundice and cough problems. }\end{array}$ & $\begin{array}{l}\text { Khan and Khatoon, } \\
\text { 2007. Gilgit } \\
\text { Baltistann }\end{array}$ \\
\hline
\end{tabular}

\section{Quantitative Analysis of the data Use Value}

Quantitative data about the use value of medicinally important plants were obtained calculating the relative importance of these species in the community. The use values ranged from 5.473 for Berberis lyceum to 0.125 for Pedicularis pectinata. The use values of the remaining plants were distributed between these two extreme values. Details of all the medicinal plants along with use values are given in Table 2. The higher use values might be attributed to the common distribution of medicinal plants and their use awareness of the inhabitants (Khan et al., 2011; Ullah et al., 2014).

\section{Relative Frequency of Citation}

Relative frequency of citation indicates the local importance of each species with reference to informants who mentioned these medicinal plant species. The RFC values of all 90 plant-species varied from 0.96 for Berberis lyceum to 0.03 for Aster himalaicus (Fig. 13 Table 2).

The comparatively higher RFC values may be due to easy availability of the respective plant, wide range of distribution, and frequent/multiple uses. The current results were in contradiction to Abbasi et al. (2013) for most of the plant species in common. The contradiction can be attributed to the developmental difference of the study areas and cultural differences.
The medicinal plants with high RFC might be interesting for pharmacological, biological evaluation for the validation of traditional knowledge. (Mukherjee et al., 2012). Based upon the results, the interesting medicinal plants might be subjected to phytochemical screening in the quest of new drugs candidates.

\section{Pearson Correlation Coefficient}

The Pearson Correlation Coefficient is used to find out the nature of linear relationship between RFC and UV and its numerical value was found to be 0.836 (at $\mathrm{P}$-value $<1 \%$ ). This relationship provides evidence of positive significant association between the local importance of each species and the relative importance of plants use. It reflects that greater the use of the species by the informants tends to increase the number of usable medicinal flora. If patterns across species were matching RFC and UV were positively correlated. However, the values of RFC and UV across some species were different. This variation across species was numerically calculated by $\mathrm{r}^{2}$, where by $70 \%$ variations in RFC can be elaborated by that of UV. Thus, results suggest evincing strength among these two indices (Table 4). These results are further aided by a graph drawn in the shape of scattered plot giving positive relationship between RFC and UV (Fig. 14). 


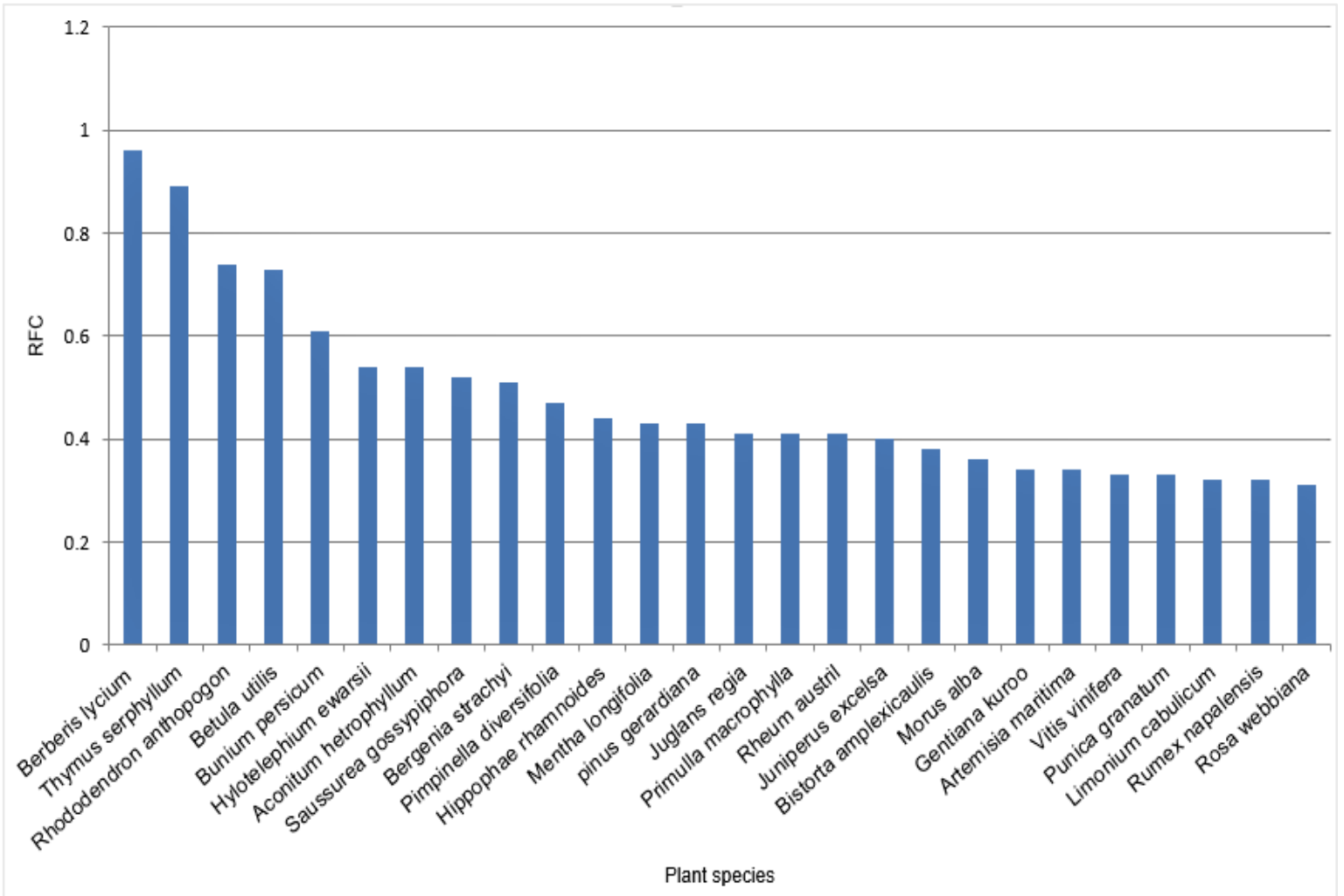

Fig. 13. Relative frequency of citation of different plants.

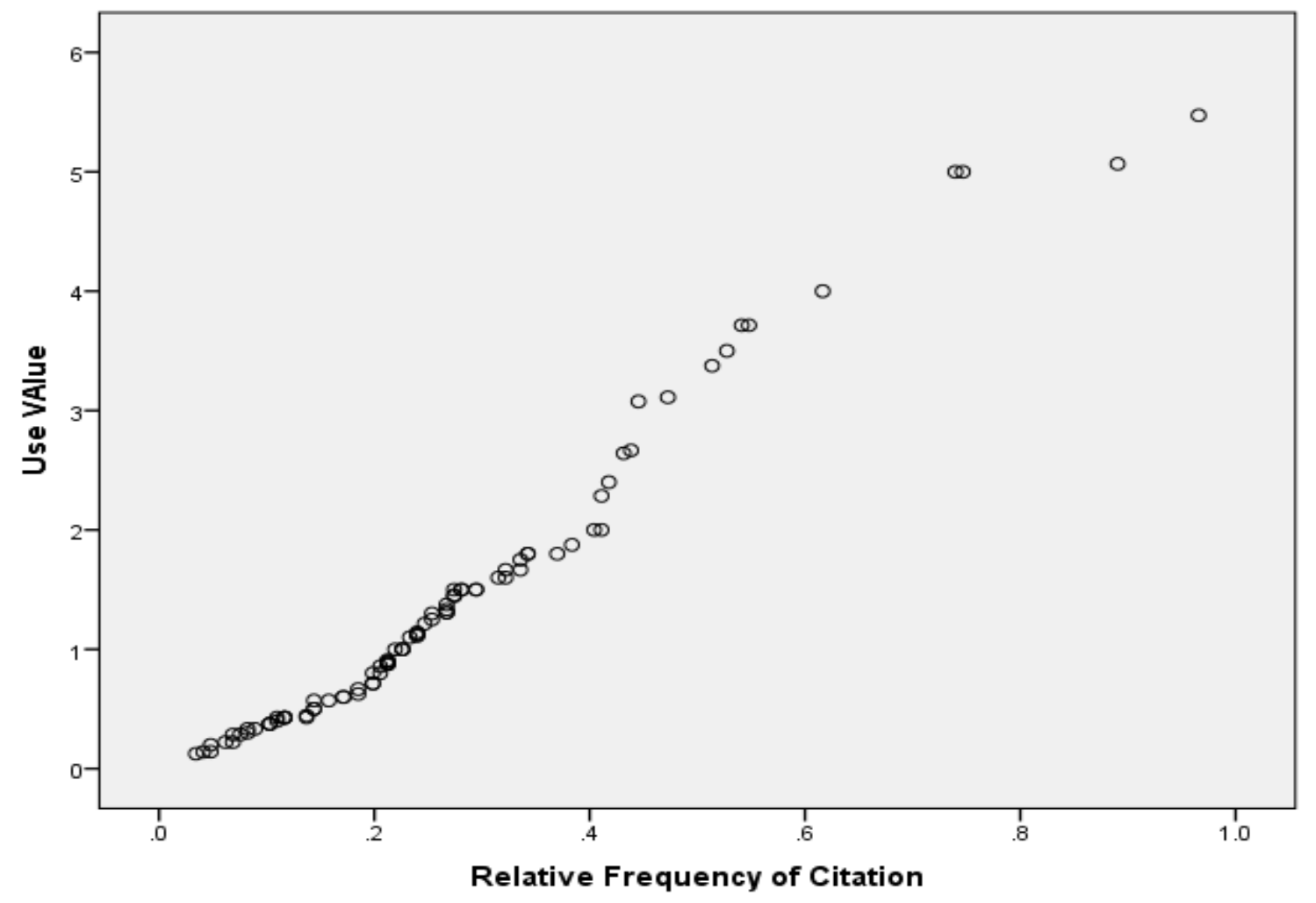

Fig. 14. Association between Relative Frequency of Citation and Use Value 
Table 4. Summary Stats for Relative Frequency of Citation (RFC) and Use Value (UV)

\begin{tabular}{|l|l|l|l|l|}
\hline & Mean & SD & Min & Max \\
\hline RFC & 0.267 & 0.179 & 0.034 & 0.965 \\
\hline UV & 1.384 & 1.208 & 0.125 & 5.47 \\
\hline \multicolumn{3}{|c|}{ Association between RFC and UV by Using } \\
Pearson Correlation Method \\
\hline R & \multicolumn{4}{|c|}{0.836} \\
\hline $\mathbf{r}^{2}$ & \multicolumn{4}{|c|}{0.699} \\
\hline
\end{tabular}

SD =Standard Deviation,

Min =Minimum, Max =Maximum

\section{Conclusions}

This study was a challenging adventure documenting valuable traditional knowledge. The results obtained included new use reports for many plants and 3 new medicinal plants reported for the first time documented from Pakistan. This indicated that further studies are clearly needed. Further, the findings of this study can broaden the foundation of herbal medicines as well as pharmacology, pharmacy, phytochemistry inviting experimental studies for the validation of traditional and isolation of compounds of therapeutic importance for the development of new drugs against challenging and complex diseases like cancer.

\section{Declarations}

Ethics approval and consent to participate:

Before conducting interviews, prior informed consent was obtained from all participants. No further ethics approval was required.

Consent for publication: This manuscript does not contain any individual person's data and further consent for publication is not required.

Availability of data and materials: The data can be obtained from the corresponding author upon request.

Funding: This was no funding available from any source to conduct this study. The authors himself bear all the expenditures to conduct the fieldwork and collection of data.

Competing financial interests: The authors declare that they have no competing financial interest.

Author contributions: RW, ZRM, NIR and designed the study, conducted the field work, collected the data and RW and KR developed the manuscript. $R Q$ and RWB improved the manuscript and restructured the data analysis. All authors read, corrected and approved the final manuscript

\section{Acknowledgments}

The authors gratefully acknowledge the local community of the study area for sharing their traditional medicinal knowledge.

\section{Literature cited}

Abbas Q, Khan SW, Khatoon S, Hussain SA, Najamul HS, Hussain A, Qureshi R, Hussain I. 2014. Floristic biodiversity and traditional uses of medicinal plants of Haramosh Valley Central Karakoram National Park of Gilgit district, Gilgit-Baltistan, Pakistan. Journal of Biodiversity and Environmental Sciences. 5, 75-86.

Abbasi AM, Khan MA, Shah MH, Shah MM, Pervez A, Ahmad M. 2013. Ethnobotanical appraisal and cultural values of medicinally important wild edible vegetables of Lesser Himalayas-Pakistan. Journal of Ethnobiology and Ethnomedicine. 9: 1-7.

Afifi FU, Abu-Irmaileh B. 2000. Herbal medicine in Jordan with special emphasis on less commonly used medicinal herbs. Journal of Ethnopharmacology. 72: 101-110.

Afzal S, Mir AK, Tayyaba, S. 2009. Ethno-botanical studies from Northern Pakistan. Journal of Ayub Medical College Abbottabad. 21: 52-57.

Ahmad K, Pieroni, A. 2016. Folk knowledge of wild food plants among the tribal communities of Thakhte-Sulaiman Hills, North-West Pakistan. Journal of Ethnobiology and Ethnomedicine. 12(1):17.

Ahmad U, Wali R, llyas N, Batool N, Gul R. 2015. Evaluation of compost with different NPK level on pea plant under drought stress. Pure and Applied biology. 4(2), 261-267.

Amara U, Mashwani ZR, Khan A, Laraib S, Wali R, Sarwar U, Ain QT, Shakeel S, Rahimullah, Sohail. 2017. Conservation status and therapeutic potential of Saussurea lappa: An overview. American Journal of Plant Sciences. 8, 602-614.

Awan MR, Jamal Z, Khan A. 2013. Ethno-botanical studies of economically important plants from mountainous region of Gilgit-Baltistan, Pakistan. Science, Technology and Development. 32(4), 308318.

Ayyanar M, Ignacimuthu S. 2005. Traditional knowledge of kani tribals in Kouthalai of Tirunelveli hills, Tamil Nadu, India. Indian Journal Ethnopharmacolpgy. 102(2), 246-255. 
Bano A, Ahmad M, Hadda TB, Saboor A, Sultana S, Zafar M, Ashraf MA, 2014. Quantitative ethnomedicinal study of plants used in the skardu valley at high altitude of Karakoram-Himalayan range, Pakistan. Journal of ethnobiology and ethnomedicine, 10(1), 43.

Bano A, Ahmad M, Khan M.A, Zafar M, Sultana S, Ullah Z, 2012. Pollen morphology of four endemic species of Pedicularis L. from alpine zone of the Deosai Plateau, Himalayan range. Bangladesh Journal of Plant Taxonomy. 19(1), 1-5.

Bano A, Ahmad M, Zafar M, Sultana S, Rashid S Khan, MA. 2014. Ethnomedicinal knowledge of the most commonly used plants from Deosai Plateau, Western Himalayas, Gilgit Baltistan, Pakistan. Journal Ethnopharmacology. 155(2), 1046-1052.

Benítez G, González-Tejero MR, Molero-Mesa J. 2010. Pharmaceutical ethnobotany in the western part of Granada province (southern Spain): Ethnopharmacological synthesis. Journal of Ethnopharmacology. 129(1), 87-105.

Bibi Y, Nisa S, Chaudhary FM, Zia M. 2011. Antibacterial activity of some selected medicinal plants of Pakistan. BMC Complementary and Alternative Medicine. 11(1), 52.

Fahad S, Bano A. 2012. Ethnobotanical and physiological studies of some endangered plant species collected from two different altitudes in Gilgit Baltistan. Pakistan Journal of Botany. 44, 165-170.

Ghorbani A. 2005. Studies on pharmaceutical ethnobotany in the region of Turkmen Sahra, north of Iran:(Part 1): General results. Journal of Ethnopharmacology. 102(1), 58-68.

Giday M, Asfaw Z, Woldu Z, Teklehaymanot T. 2009. Medicinal plant knowledge of the Bench ethnic group of Ethiopia: an ethnobotanical investigation. Journal of Ethnobiology and Ethnomedicine. 5(1), 34.

Hamayun M. 2005. Ethnobotanical profile of Utror and Gabral valleys, district Swat, Pakistan. Ethnobotonical Leaflets. 1, 9.

Haq. F, 2012. The Ethno Botanical Uses of Medicinal Plants of Allai Valley, Western Himalaya Pakistan. International Journal of Plant Research. 2(1): 21-34.

Hussain I, Bano A, Ullah F. 2011. Traditional drug therapies from various medicinal plants of central karakoram national park, Gilgit-Baltistan Pakistan. Pakistan Journal of Botany. 43, 79-84.

Jalal JS, Kumar P. 2006. Some Ethno-Medicinal Plants of Gori River Basin, Kumaon Himalaya. Flora. 12(2): 292-294.

Kala CP. 2005. Indigenous Uses, Population Density, and Conservation of Threatened Medicinal
Plants in Protected Areas of the Indian Himalayas. Conservation Biology. 19(2), 368-378.

Khan B, Abdukadir A, Qureshi R, Mustafa G. 2011. Medicinal uses of plants by the inhabitants of Khunjerab National Park, Gilgit, Pakistan. Pakistan Journal of Botany. 43(5), 2301-2310.

Khan SR, 1997. Micro case study and action plan for fairy meadows: International Centre for Integrated Mountain Development.

Khan SW, Khatoon S. 2007. Ethnobotanical studies on useful trees and shrubs of Haramosh and Bugrote valleys In Gilgit Notheren areas of Pakistan. Pakistan Journal of Botany. 39(3), 699-710.

Khan SW, Khatoon S. 2008. Ethnobotanical studies on some useful herbs of Haramosh and Bugrote valleys in Gilgit, northern areas of Pakistan. Pakistan Journal of Botany. 40(1): 43-58.

Khan T, Khan IA, Rehman A, Alam J, Ali S. 2013. Exploration of near-extinct folk wisdom on medicinally important plants from Shinaki Valley Hunza, Pakistan. International Journal of Biosciences. 3(10), 180-186.

Manandhar N.P, 2002. Plants and People of Nepal. Timbre Press, Oregon, USA.

Muhammad G, Khan MZ, Hussain MH, lqbal Z, Iqbal M, Athar M. 2005. Ethnoveterinary practices of owners of pneumatic-cart pulling camels in Faisalabad City (Pakistan). Journal of Ethnopharmacology. 97(2), 241-246.

Mukherjee PK, Nema NK, Venkatesh P, Debnath PK. 2012. Changing scenario for promotion and development of Ayurveda-way forward. Journal of Ethnopharmacology 143(2), 424-434.

Nautiyal S, Makhuri RK, Rao KS, Ssaxena KG. 2008. Medicinal Plant Resources in Nanda Devi Biosphere Reserve in the Central Himalayas. Journal of Herbs, Spices and Medicinal Plants. 8(4) 47-64.

Pant SR, Panta IR. 2004. Indigenous knowledge on medicinal plants in Bhagawati village, Darchula, Nepal. Botanica Orientalis: Journal of Plant Sciences. 4(1):79-81.

Phillips OL, Hall P, Gentry AH, Sawyer SA. Vasquez R.1994. Dynamics and species richness of tropical rain forests. Proceedings of the National Academy of Sciences. 91(7), 2805-2809.

Pieroni A, Guuisti ME. 2009. Alpine ethnobotany in Italy: traditional knowledge of gastronomic and medicinal plants among the Occitans of the upper Varaita valley, Piedmont. Journal of Ethnobiology and Ethnomedicine. 5(1):32.

Pieroni A, Quave C, Nebel S, Heinrich M. 2002. Ethnopharmacy of the ethnic Albanians of Northern Basilicata, Italy. Fitoterapia. 73: 217-241. 
Prabhu S, Vijayakumar S, Yabesh JEM. Ravichandran K, Sakthivel B. 2014. Documentation and quantitative analysis of the local knowledge on medicinal plants in Kalrayan hills of Villupuram district, Tamil Nadu, India. Journal of Ethnopharmacology. 157, 7-20.

Qureshi RA, Ghufran MA, Sultana KN, Ashraf M, Khan. AG. 2007. Ethnomedicinal studies of medicinal plants of Gilgit District and surrounding areas. Ethnobotany Research and Applications. 5, 115-122.

Rehman K, Mashwani ZR, Khan MA, Ullah Z, Chaudhary HJ. 2015. An ethnobotanical perspective of traditional medicinal plants from the Khattak tribe of Chonthra Karak, Pakistan. Journal of Ethnopharmacology. 165, 251-259.

Saric-Kundali B, Dobes C, Klatte-Asselmeyer V, Saukel J. 2010. Ethnobotanical study on Medicinal use of wild and cultivated plants in Middle, South and West Bosnia and Herzegovina. Journal of Ethnopharmacology. 131: 33-55.

Shedayi AA, Gulshan B. 2012. Ethnomedicinal uses of plant resources in Gilgit-Baltistan of Pakistan. Journal of Medicinal Plants Research. 6(29), 45404549.

Shedayi AA, Xu M, Gulraiz B. 2014. Traditional medicinal uses of plants in Gilgit-Baltistan, Pakistan. Journal of Medicinal Plants Research. 8(30), 9921004.

Shinwari Zk, Gilani SS. 2003. Sustainable harvest of medicinal plants at Bulashbar Nullah, Astore (northern Pakistan). Journal of Ethnopharmacology. 84(2), 289-298.

Shinwari Zk, Qaiser M. 2011. Efforts on conservation and sustainable use of medicinal plants of Pakistan. Pakistan Journal of Botany. 43(1), 5-10.

Shinwari Zk, Salima M, Faisal R, Huda S, Asrar M. 2013. Biological screening of Indigenous knowledge based plants used in Diarrheal treatment. Pakistan Journal of Botany. 45(4), 1375-1382.

Tardío J, Pardo-de-Santayana M. 2008. Cultural importance indices: a comparative analysis based on the useful wild plants of Southern Cantabria (Northern Spain) 1. Economic Botany. 62(1), 24-39.

Telkes N. 2011. Horsetail: Cultivating and Building a New Relationship. LMT and Practicing Herbalist (RH). American Herbalists Guild. The Wildflower School of Botanical Medicine www.nicoletelkes.com.

Thapa S. 2001. Documentation of traditional uses of plants by Tharu community around Royal SuklaPhanta Wildlife Reserve, Far west Nepal. Central Department of Botany, Tribhuvan University, Kathmandu, Nepal. 61.
Ullah M, Khan MU, Mahmood A, Malik RN, Hussain M, Wazir SM, Daud M, Shinwari ZK, 2013. An ethnobotanical survey of indigenous medicinal plants in Wana district south Waziristan agency, Pakistan. Journal of Ethnopharmacology. 150(3), 918-924.

Ullah S, Khan, MR, Shah NA, Shah SA, Majid M, Farooq MA. 2014. Ethnomedicinal plant use value in the Lakki Marwat District of Pakistan. Journal of Ethnopharmacology. 158, 412-422.

Umadevi M, Kumar, KPS, Bhowmik D, Duraivel S. 2013. Traditionally used anticancer herbs in India. Journal of Medicinal Plants Studies. 1(3), 56-74.

Uniyal SK, Singh KN, Jamwal P, Lal B. 2006. Traditional use of medicinal plants among the tribal communities of Chhota Bhangal, Western Himalaya. Journal of Ethnobiology and Ethnomedicine. 2(1):14 Vitalini S, Iriti, M, Puricelli, C, Ciuchi, D, Segale, A, Fico, G. 2013. Traditional knowledge on medicinal and food plants used in Val San Giacomo (Sondrio, Italy)_An alpine ethnobotanical study. Journal of Ethnopharmacology. 145(2), 517-529.

Wagay, N.A., 2016. Ethnobotany from North Kashmir: Life Science Leaflets. 80: 38-60.

Wazir SM, Dasti, AA, Shah J. 2004. Common medicinal plants of chapursan valley, Gojal II, GilgitPakistan. Journal of Research (Science) Bahauddin Zakariya University, Multan, Pakistan. 15, 41-43.

Yabesh JEM, Prabhu S, Vijayakumar S. 2014. An ethnobotanical study of medicinal plants used by traditional healers in silent valley of Kerala, India. Journal of Ethnopharmacology. 154(3), 774-789.

Zheng X, Wang W, Piao H, Xu W, Shi H, Zhao, C. 2013. The Genus Gnaphalium L. (Compositae): Phytochemical and Pharmacological Characteristics. Molecules. 18(7), 8298-8318.

of some plants of Chagharzai valley, district Buner, Pakistan. Pakistan Journal of Botany. 43(3):14451452.

Shinwari MI, Khan MA. 2000. Folk use of medicinal herbs of Margalla hills national park, Islamabad. Journal of Ethnopharmacology. 69(1):45-56.

Shinwari ZK, Gilani SS. 2003. Sustainable harvest of medicinal plants at Bulashbar Nullah, Astore (northern Pakistan). Journal of Ethnopharmacology. 84(2):289-298.

Street RA, Prinsloo G. 2013. Commercially important medicinal plants of South Africa: a review. Journal of Chemistry. 1-16. doi:10.1155/2013/205048.

Tabuti JRS, Dhillion SS, Lye KA. 2004. The status of wild food plants in Bulamogi County, 
Uganda. International Journal of Food Sciences and Nutrition. 55(6):485-498.

Tareen NM, Saeed UR, Shinwari ZK, Bibi T. 2016. Ethnomedicinal utilization of wild edible vegetables in district Harnai of Balochistan province, Pakistan. Pakistan Journal of Botany. 48(3):1159-1171.

Tariq A, Mussarat S, Adnan M, Abd-Allah EF, Hashem A, Alqarawi AA, Ullah R. 2015. Ethnomedicinal evaluation of medicinal plants used against gastrointestinal complaints. Biomed Research International. 2015: 1-14. Article ID 892947.

Haq UI, Hussain M. 1993. Medicinal plants of Mansehra. Hamdard Medicine Journal. 36(3):63100.

Ullah M, Khan MU, Mahmood A, Malik RN, Hussain M, Wazir SM, Daud M, Shinwari ZK. 2013. An ethnobotanical survey of indigenous medicinal plants in Wana district South Waziristan agency, Pakistan. Journal of Ethnopharmacology. 150(3):918-924.

Uniyal SK, Singh KN, Jamwal P, Lal B. 2006. Traditional use of medicinal plants among the tribal communities of Chhota Bhangal, Western Himalaya. Journal of Ethnobiology and Ethnomedicine. 2(1):14.

Upadhyay B, Singh KP, Kumar A, 2010. Pharmacognostical and antibacterial studies of different extracts of Euphorbia hirta L. Journal of Phytology. 2(6): 55-60.

WHO. 2014. World Health Statistics. WHO Press, Switzerland, 2014.

Zheng XL, Xing FW. 2009. Ethnobotanical study on medicinal plants around Mt. Yinggeling, Hainan Island, China. Journal of Ethnopharmacology. 124(2):197-210. 